
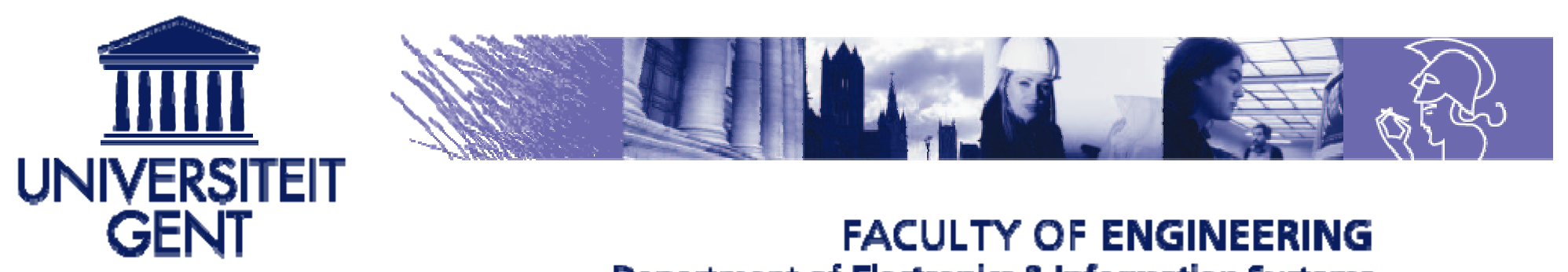

FACULTY OF ENGINEERING

Department of Electronics \& Information Systems

\title{
A Fixed-Angle Heat Spreading Model for Dynamic Thermal Characterization of Rear-Cooled Substrates
}

\section{Bjorn Vermeersch}

Seminar 'Physical Electronics'

\section{ELIS - 5 April 2007}




\section{Outline}

- Introduction

- Model definition

- Exact calculations

- Results

- Anisotropic substrates

- Conclusions 


\section{Introduction}

Problem formulation

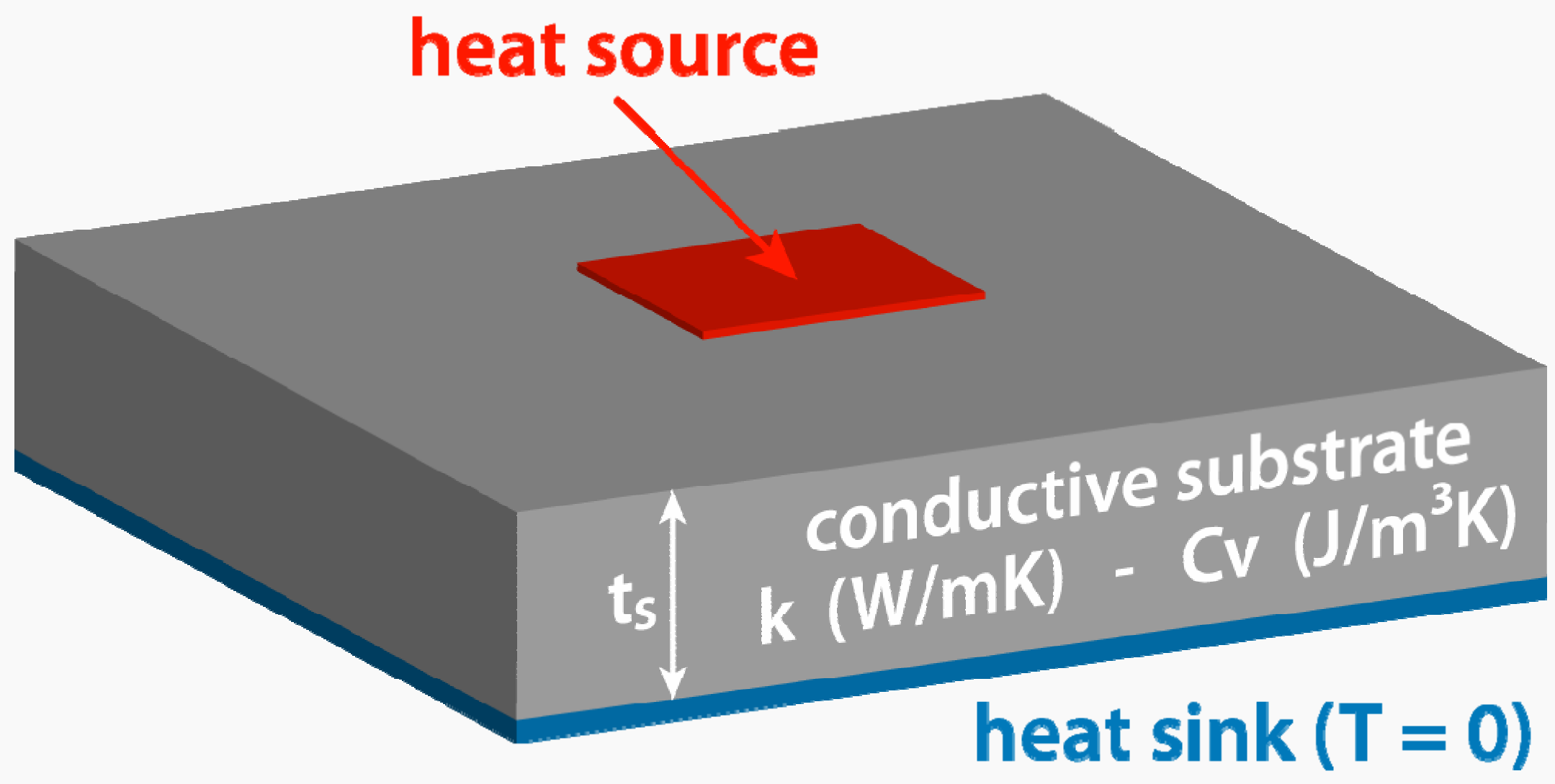

? Dynamic behaviour 


\section{Introduction}

Thermal engineering

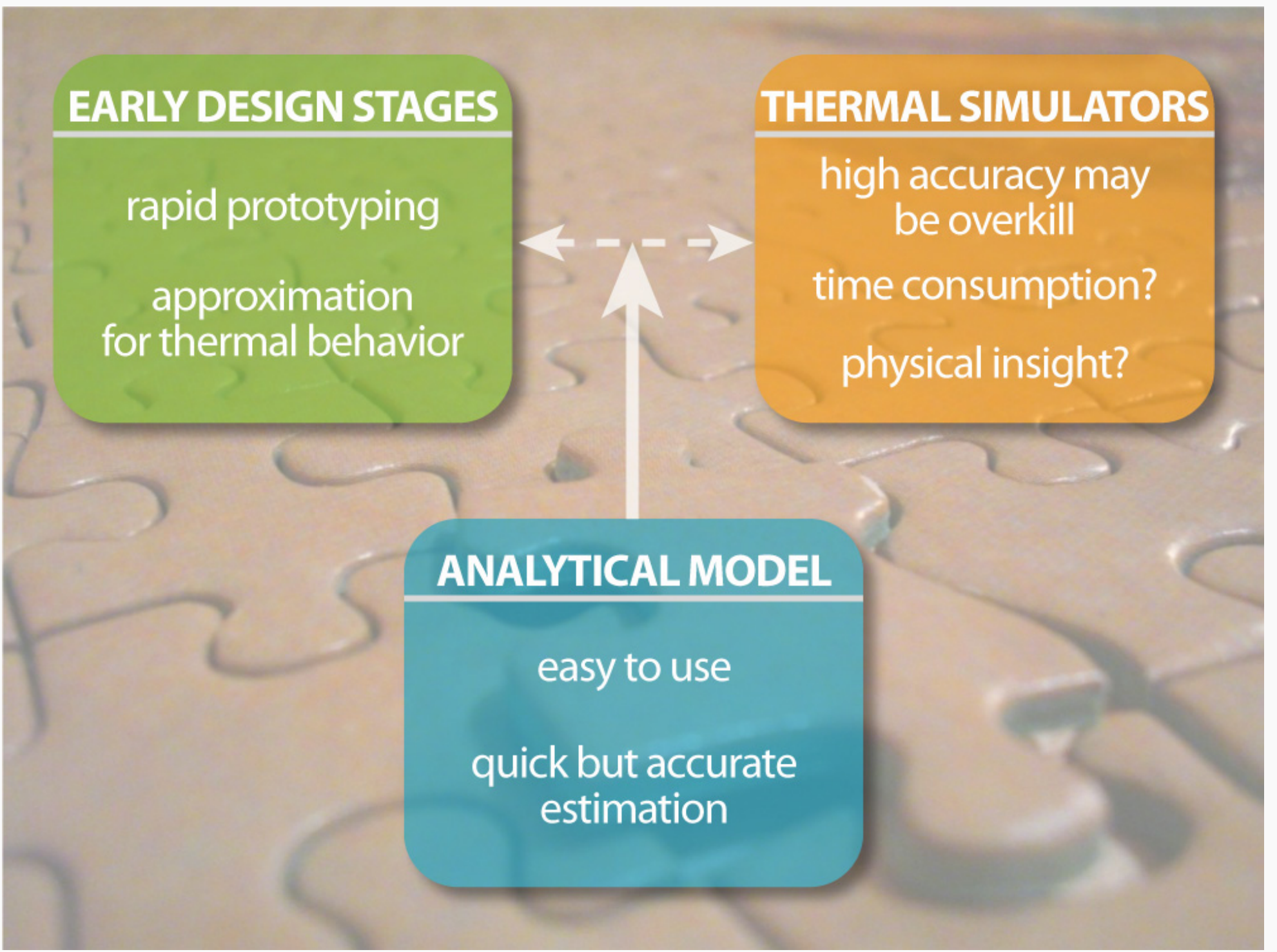




\section{Introduction Steady state fixed-angle models}

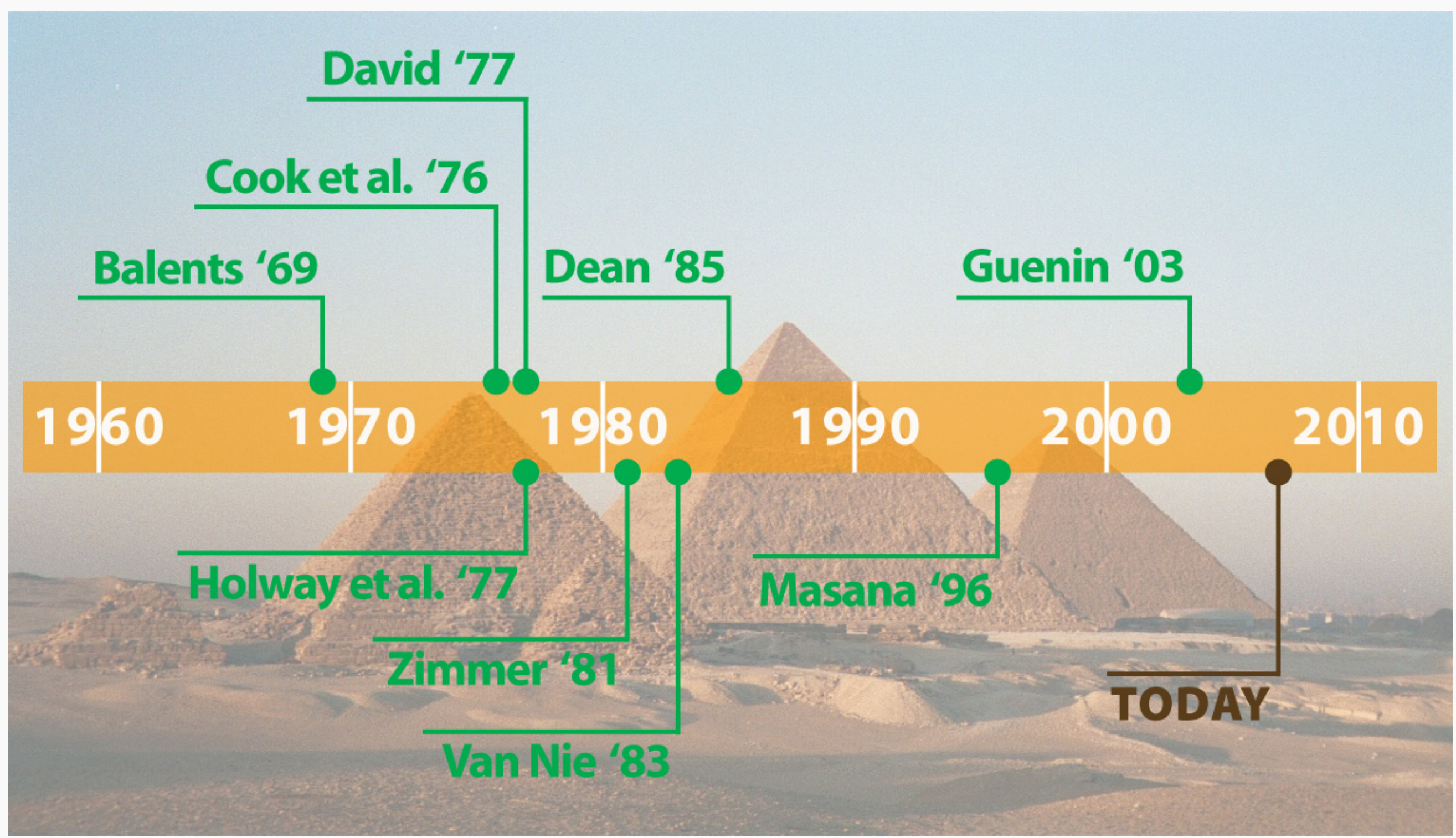




\section{Introduction}

How does a fixed-angle model look like?

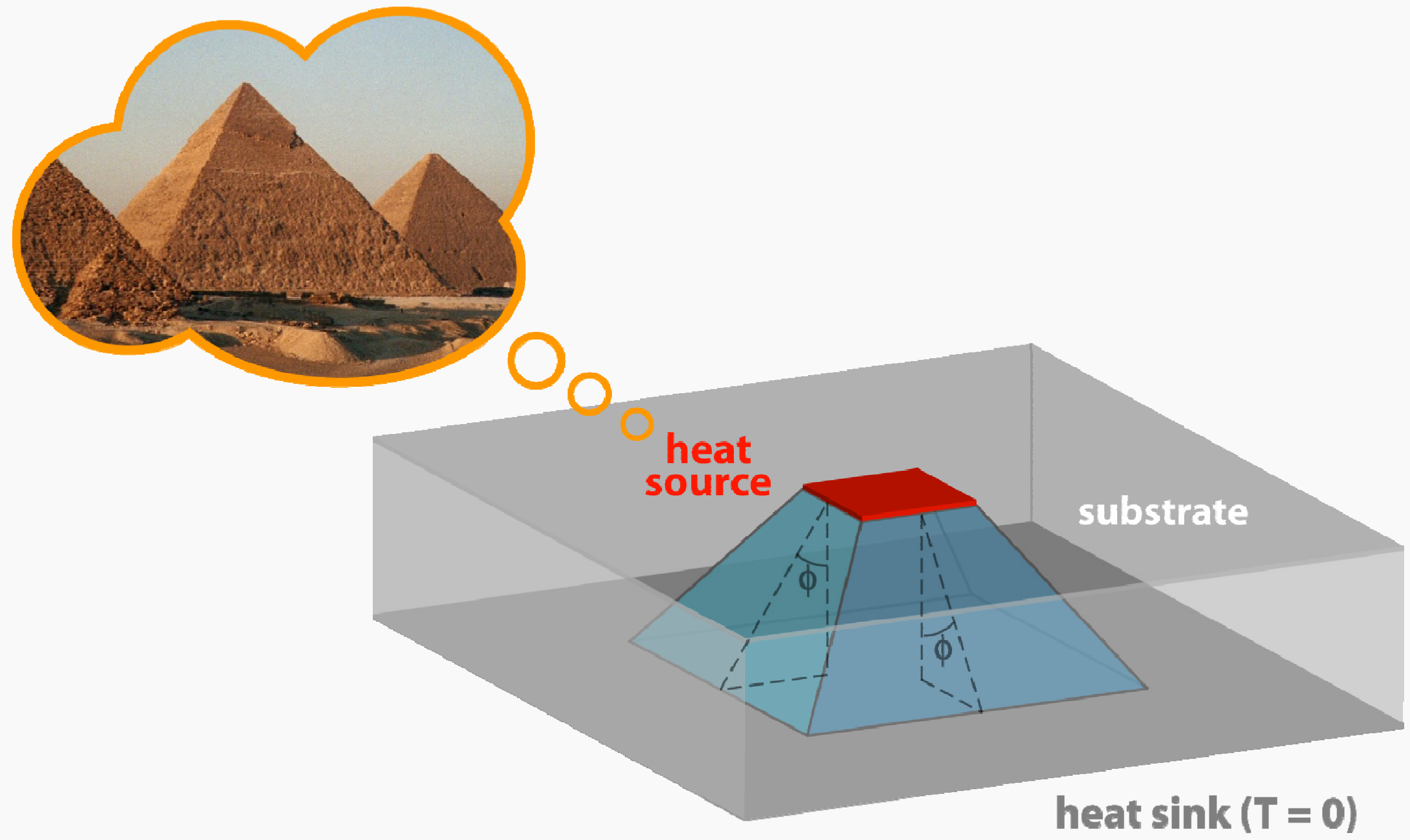




\section{Introduction}

\section{Literature overview}

\begin{tabular}{|c|c|c|c|}
\hline REFERENCE & $\begin{array}{c}\text { SPREADING } \\
\text { ANGLE }\end{array}$ & HEAT SOURCE & $\begin{array}{c}\text { SUBSTRATE } \\
\text { LAYERS }\end{array}$ \\
\hline Balents'69 & $45^{\circ}$ & $\square$ & single \\
\hline Cook'76 & $45^{\circ}$ & $\square$ multi & multi \\
\hline David'77 & $45^{\circ}, 32.5^{\circ}$ & $\square$ & single \\
\hline Holway'77 & $45^{\circ}$ & & multi \\
\hline Zimmer'81 & $32.5^{\circ}$ & $\square$ & single \\
\hline Van Nie'83 & geo & & single \\
\hline Dean'85 & $45^{\circ}$ & multi $\square$ & single \\
\hline Masana'96 & geo & $\begin{array}{c}\text { limited in } \\
\text { lateral dir. }\end{array}$ \\
\hline Guenin '03 & $45^{\circ}$ & $\square$ & single \\
\hline
\end{tabular}

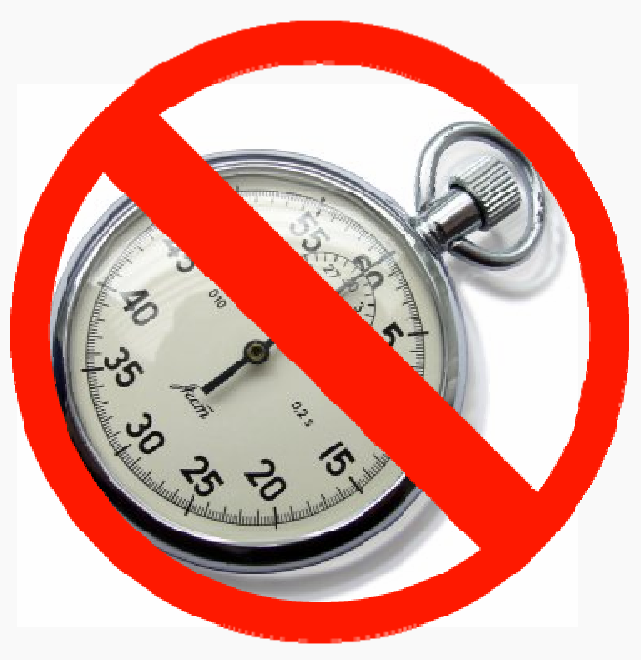

"The $45^{\circ}$ heat spreading angle: an urban legend?" 


\section{Outline}

- Introduction

- Model definition

- Exact calculations

- Results

- Anisotropic substrates

- Conclusions 


\section{Model definition}

Fixed-angle heat spreading

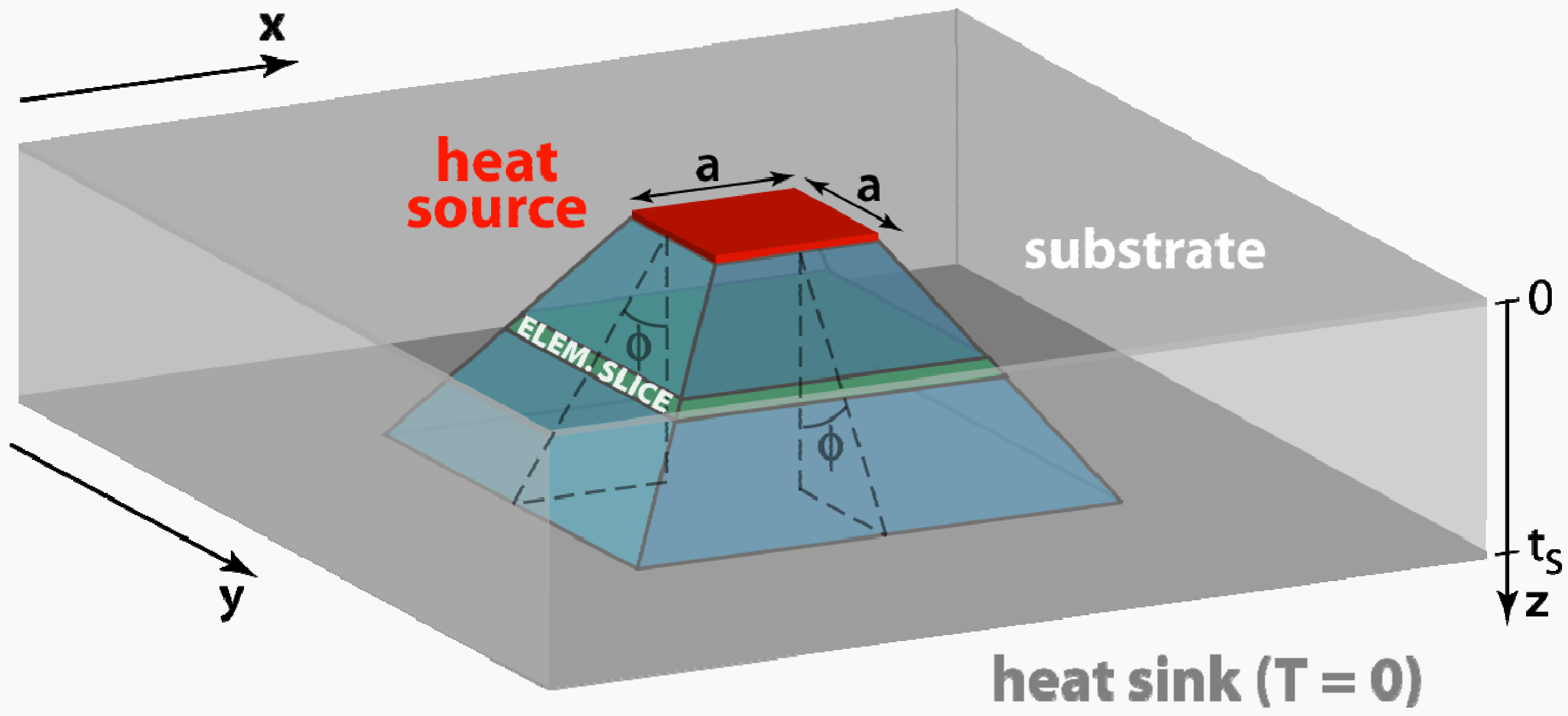




\section{Model definition}

Electrothermal analogy
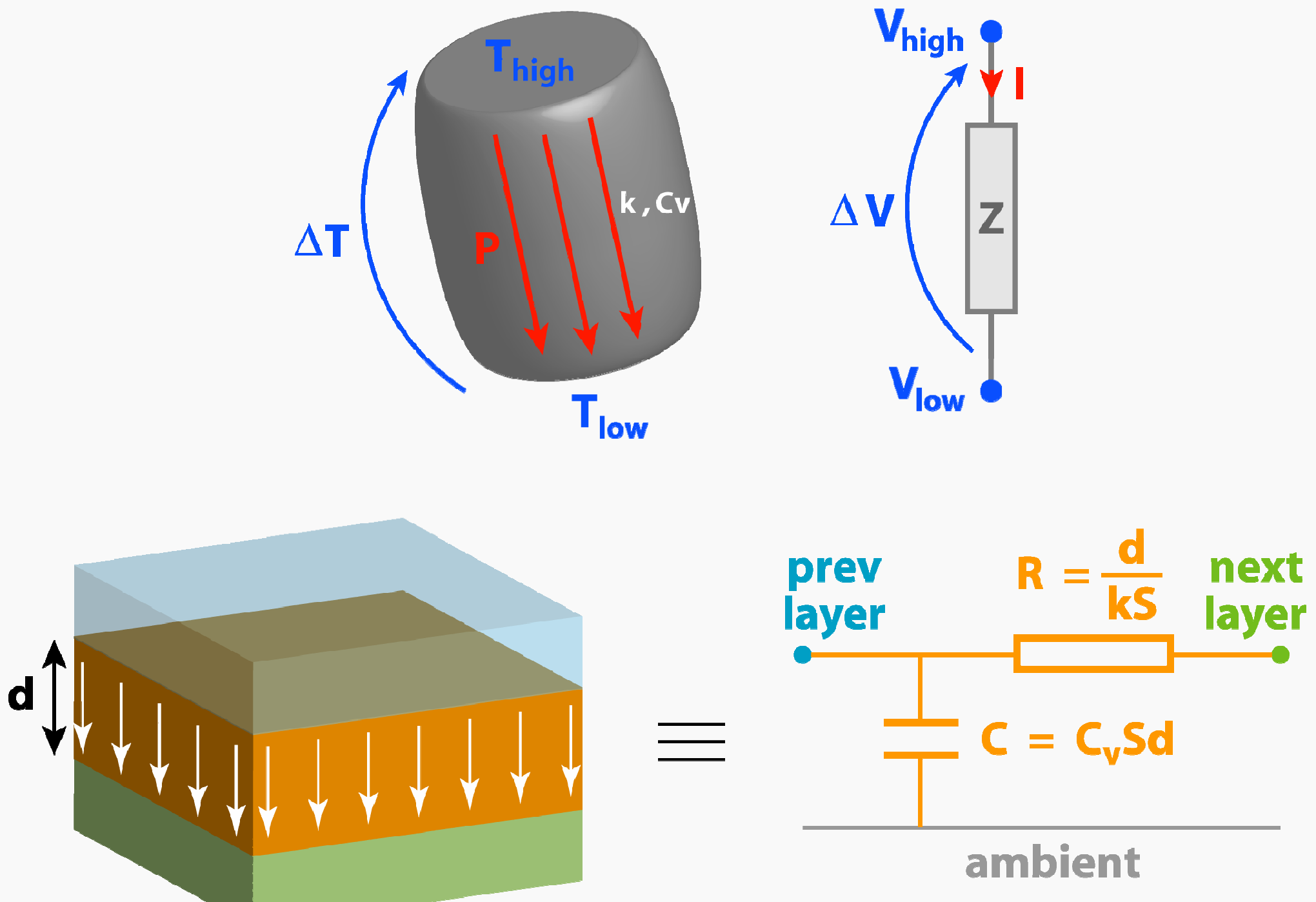


\section{Model definition}

Equivalent distributed network
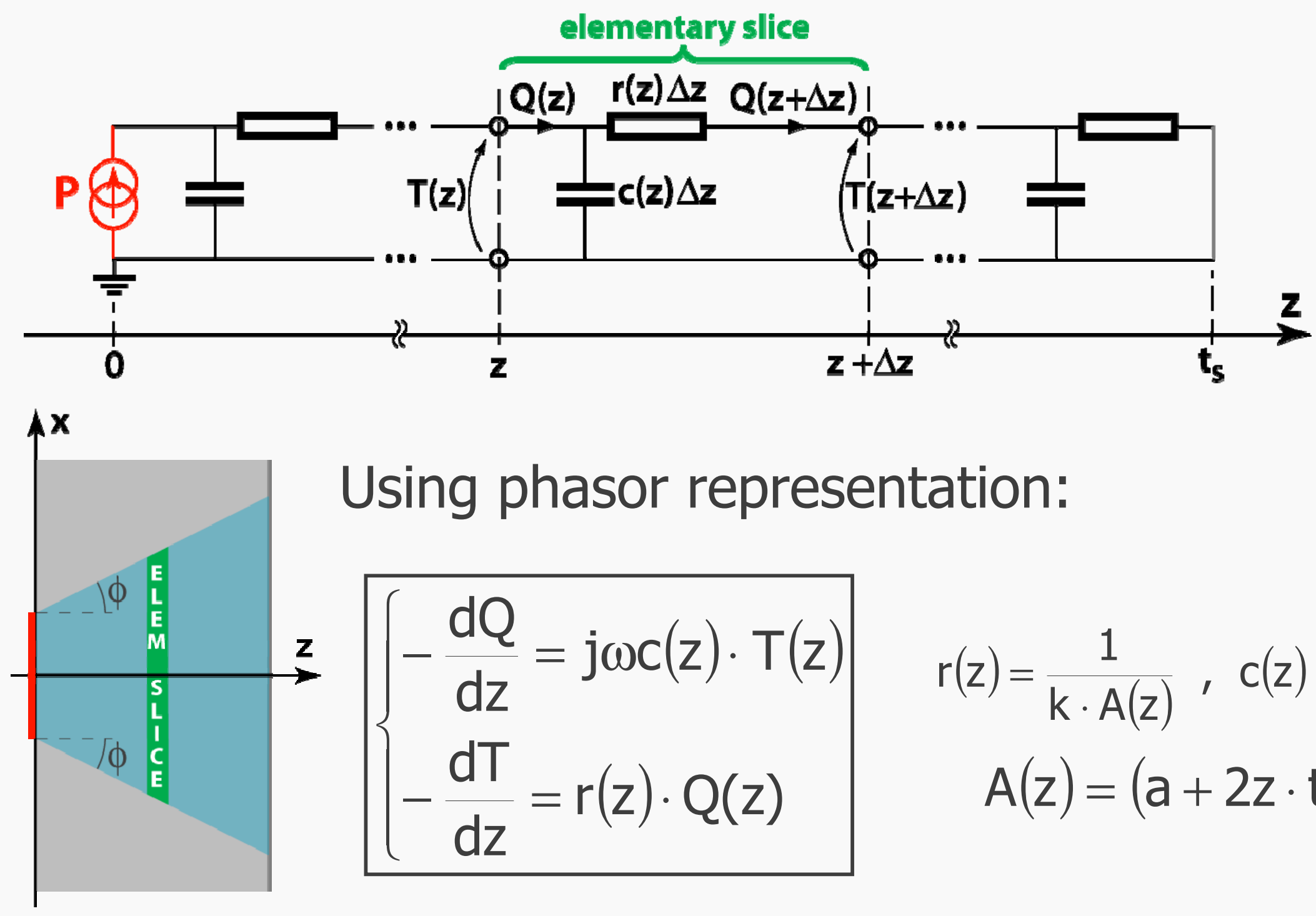

Using phasor representation:

$$
\rightarrow \begin{array}{lc}
\mathbf{z}-\frac{d Q}{d z}=j \omega c(z) \cdot T(z) & r(z)=\frac{1}{k \cdot A(z)}, c(z)=C_{v} \cdot A(z) \\
-\frac{d T}{d z}=r(z) \cdot Q(z) & A(z)=(a+2 z \cdot \tan \phi)^{2}
\end{array}
$$




\section{Model definition}

\section{Differential equation}

$\left\{\begin{array}{l}-\frac{d Q}{d z}=j \omega c(z) \cdot T(z) \\ -\frac{d T}{d z}=r(z) \cdot Q(z)\end{array} \rightarrow \begin{array}{l}\frac{d^{2} T}{d z^{2}}+\frac{2 \alpha}{1+\alpha z} \frac{d T}{d z}-\gamma T(z)=0 \\ \quad \begin{array}{c}\alpha=\frac{2 \tan \phi}{a} \\ \gamma=\frac{j \omega C_{v}}{k}\end{array}\end{array}\right.$

Substitution: $\quad \mathrm{T}(\mathrm{z})=\frac{\Psi(\mathrm{z})}{1+\alpha \mathrm{z}} \longrightarrow \frac{\mathrm{d}^{2} \Psi}{\mathrm{dz}^{2}}-\gamma \cdot \Psi(\mathrm{z})=0$

$$
\mathrm{T}(\mathrm{z})=\mathrm{C}_{1} \frac{\cosh (\gamma \mathrm{z})}{1+\alpha \mathrm{z}}+\mathrm{C}_{2} \frac{\sinh (\gamma \mathrm{z})}{1+\alpha \mathrm{z}}
$$

Boundary conditions: $\mathrm{Q}(0)=-\left.\frac{1}{\mathrm{r}(0)} \frac{\mathrm{dT}}{\mathrm{dx}}\right|_{\mathrm{x}=0}=\mathrm{P}, \mathrm{T}\left(\mathrm{t}_{\mathrm{s}}\right)=0$ 


\section{Model definition}

\section{Thermal impedance}

$\mathrm{T}(\mathrm{z})=\mathrm{C}_{1} \frac{\cosh (\gamma \mathrm{z})}{1+\alpha \mathrm{z}}+\mathrm{C}_{2} \frac{\sinh (\gamma \mathrm{z})}{1+\alpha \mathrm{z}} \quad \gamma=\frac{j \omega \mathrm{C}_{\mathrm{v}}}{\mathrm{k}}, \alpha=\frac{2 \tan \phi}{\mathrm{a}}$

- boundary conditions

- division by dissipated power $\mathbf{P}$

- normalization ( $\rightarrow$ model applicable for any material!)

$\tilde{Z}_{\mathrm{th}}(\widetilde{\omega})=\frac{1}{2 \lambda \operatorname{tar} \phi)+j \widetilde{\omega} \cdot \operatorname{cotanh}(j \widetilde{\omega})} \quad \mathrm{Z}_{0}=\frac{\mathrm{t}_{\mathrm{s}}}{\mathrm{ka}^{2}} \quad, \quad \omega_{0}=\frac{\mathrm{k}}{\mathrm{C}_{\mathrm{v}} \mathrm{t}_{\mathrm{s}}^{2}}$

$$
\lambda=\frac{t_{s}}{a}
$$

\section{fitting parameter}




\section{Outline}

- Introduction

- Model definition

- Exact calculations

- Results

- Anisotropic substrates

- Conclusions 


\section{Exact calculations}

Fundamental solution for 3D space

Green's function:

$$
\begin{array}{r}
G\left(\vec{r}^{\prime} \vec{r}^{\prime}\right)=\frac{1}{4 \pi k R} \exp \left(-j \frac{j \omega C_{v} R}{k}\right) \\
R=\left(x-x^{\prime}\right)^{2}+\left(y-y^{\prime}\right)^{2}+\left(z-z^{\prime}\right)^{2}
\end{array}
$$

For distributed heat source: superposition

$$
T(x, y, z)=\iiint_{\text {source }} p\left(\vec{r}^{\prime}\right) G\left(\vec{r}^{\prime} \vec{r}^{\prime}\right) d \vec{r}^{\prime}
$$

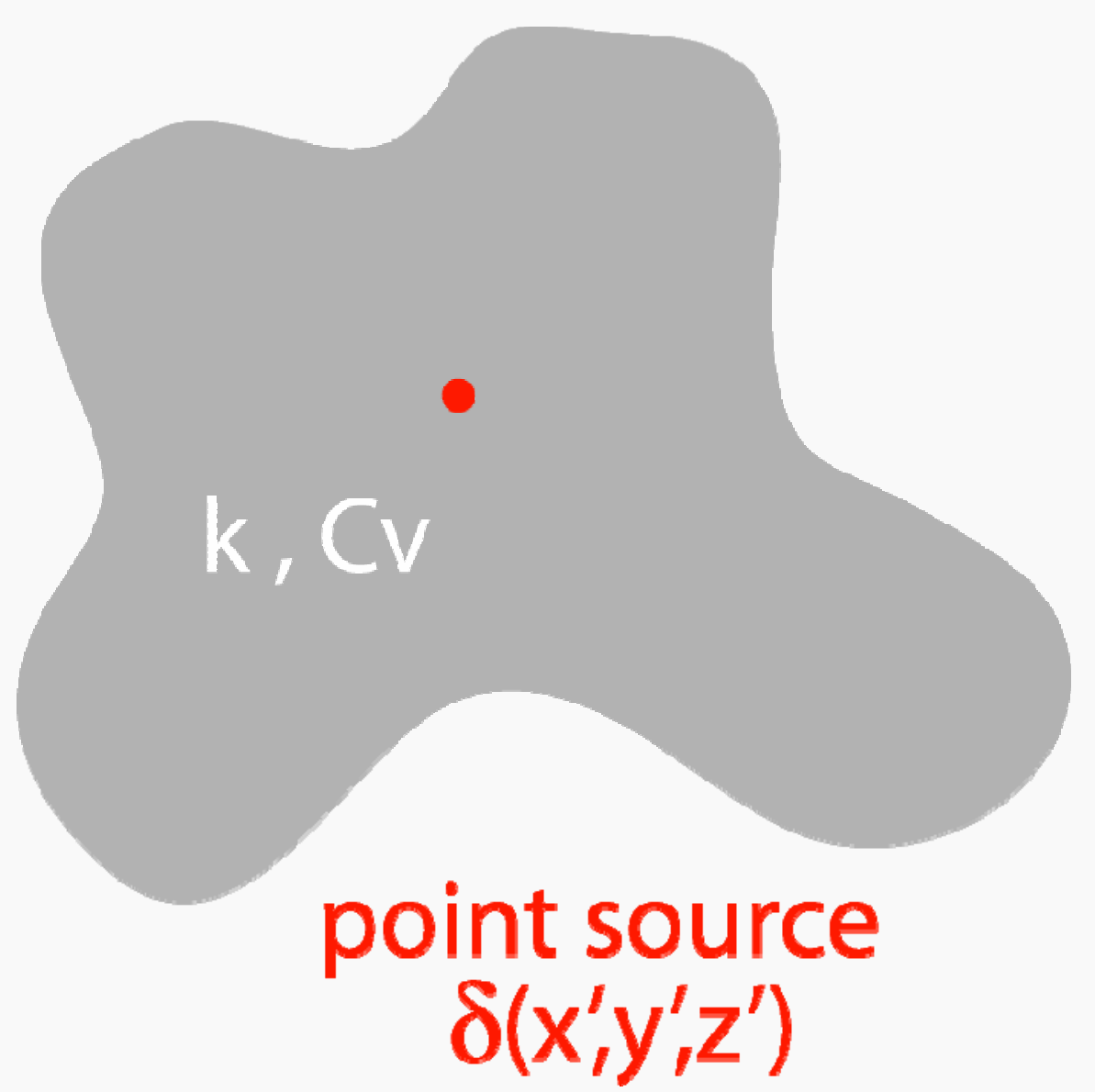




\section{Exact calculations \\ Multiple reflection technique}

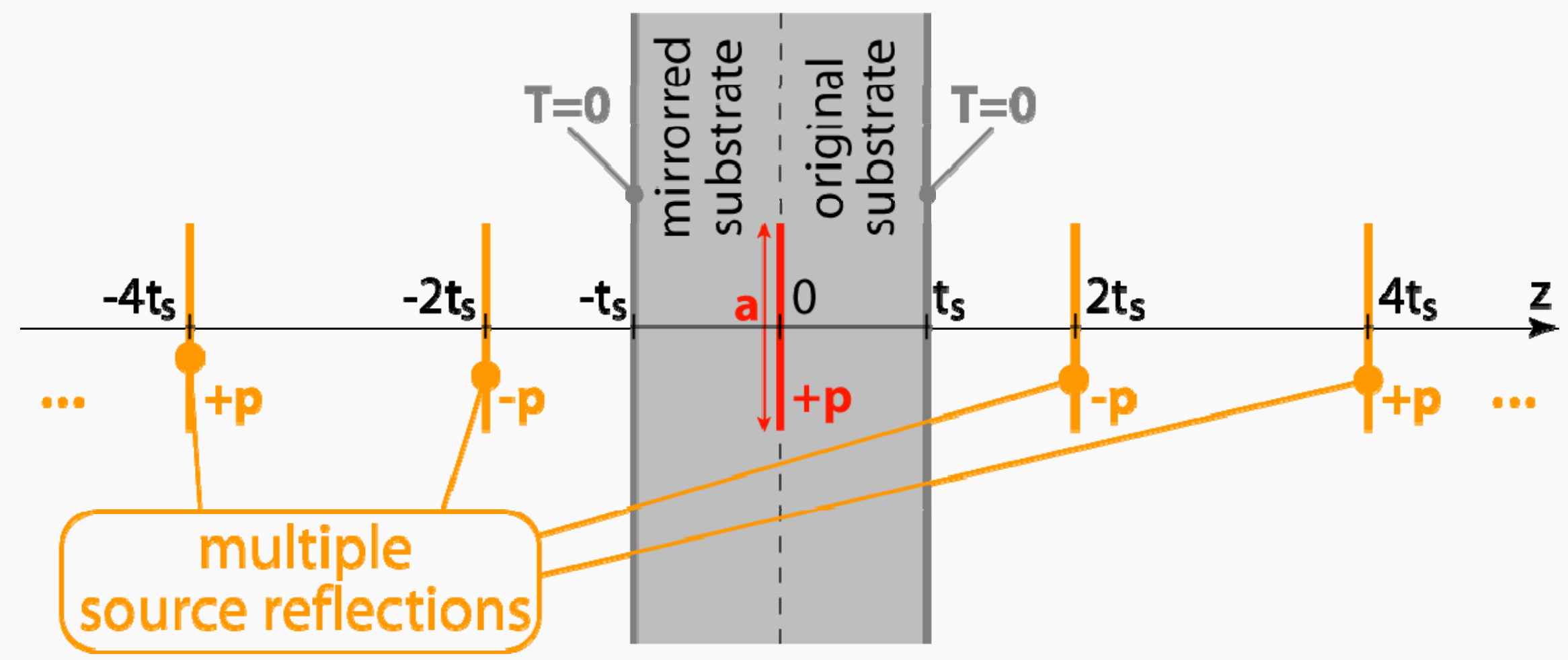

$$
\begin{aligned}
& T_{\text {source }}(x, y)=2 \frac{P}{a^{2}} \int_{-a / 2}^{a / 2} \int_{-a / 2}^{a / 2} H\left(x, x^{\prime}, y, y^{\prime}\right) d x^{\prime} d y^{\prime} \underset{\text { value }}{\stackrel{\text { average }}{\longrightarrow}} Z_{\text {th }} \\
& H=G\left(z-z^{\prime}=0\right)+2 \sum_{n=1}^{\infty}(-1)^{n} G\left(z-z^{\prime}=2 n t_{s}\right) \quad G=\text { Green's function }
\end{aligned}
$$




\section{Exact calculations}

\section{Model validation}

- calculation of sufficient number $(\mathrm{N})$ of impedance points over wide frequency range (logarithmic distribution, 10 points per decade)

- error function:

$\mathrm{e}(\phi)=\frac{1}{\mathrm{~N}} \sum_{\mathrm{i}=1}^{\mathrm{N}} \frac{\widetilde{\mathrm{Z}}_{\text {exact }}^{(\mathrm{i})}-\widetilde{\mathrm{Z}}_{\text {model }}^{(\mathrm{i})}(\phi)}{\widetilde{\mathrm{Z}}_{\text {exact }}^{(\mathrm{i})} \mid}$

(i) = evaluated at i-th frequency

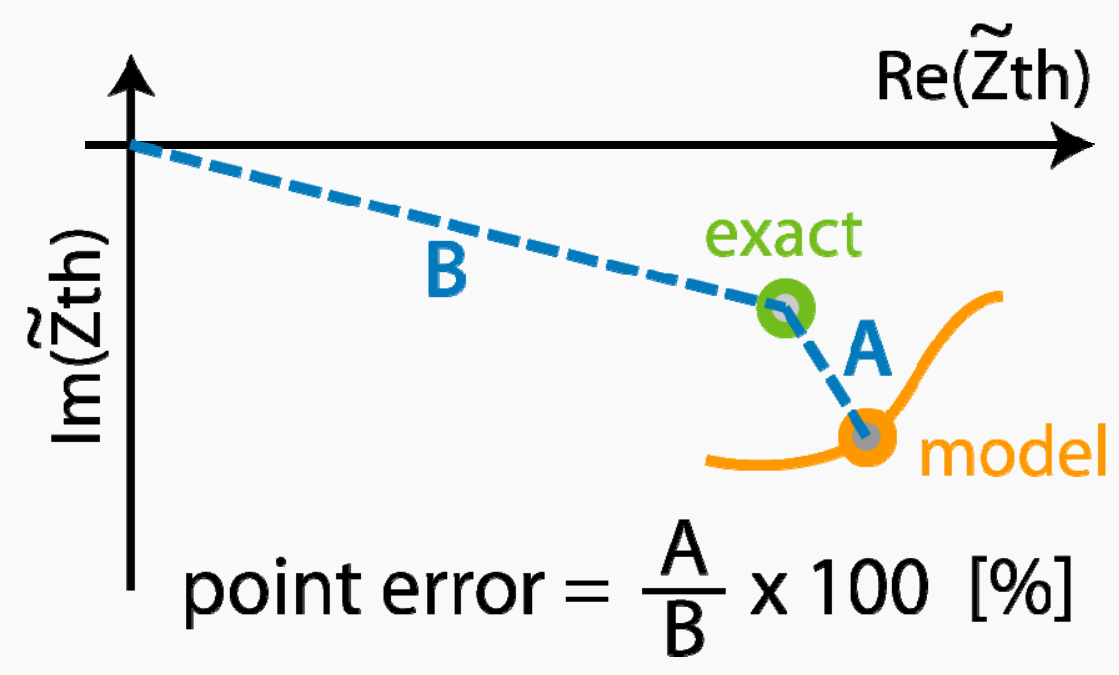




\section{Outline}

- Introduction

- Model definition

- Exact calculations

- Results

- Anisotropic substrates

- Conclusions 


\section{Results}

\section{Model validation: error curves}

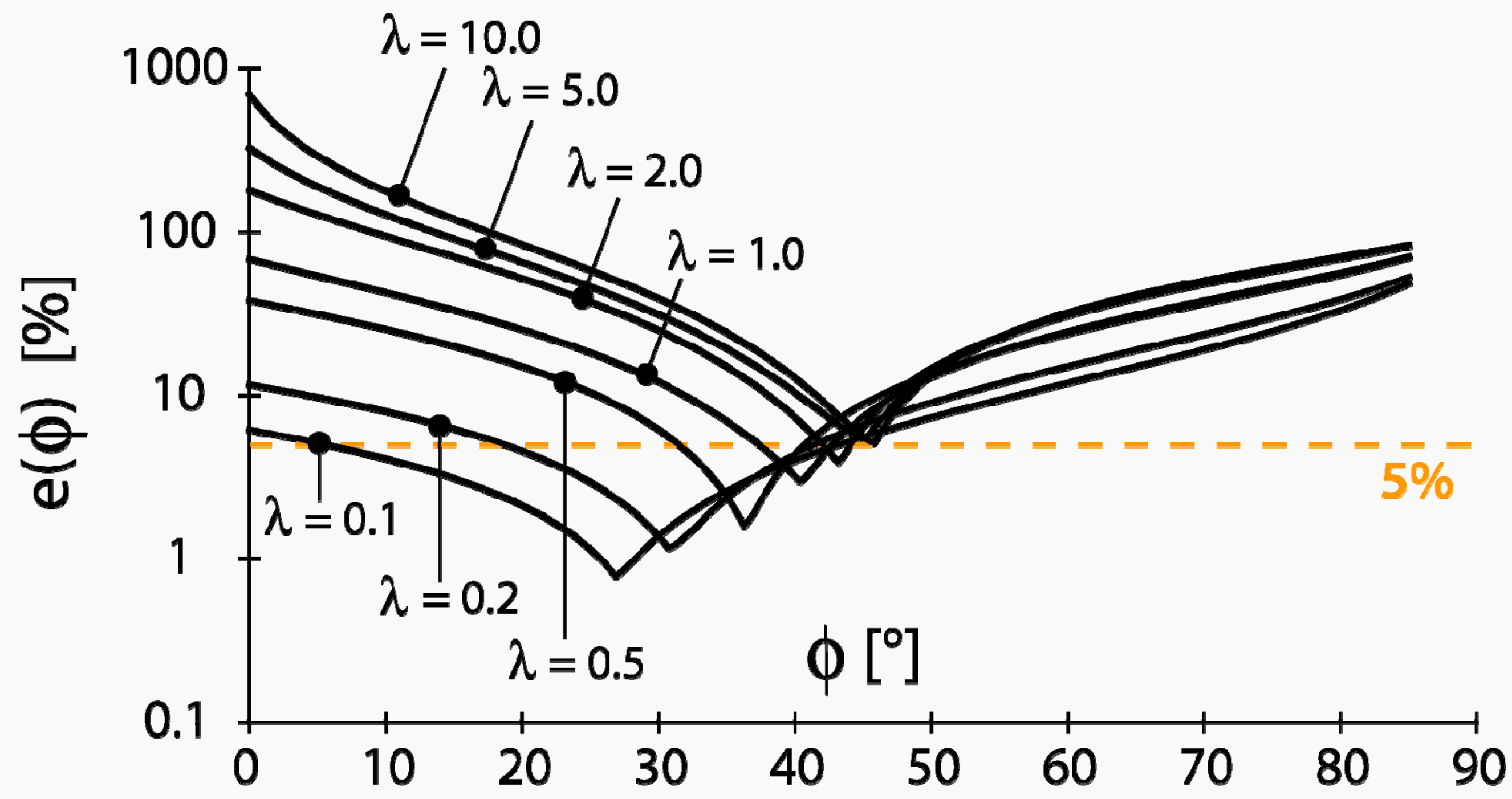




\section{Optimal spreading angle}

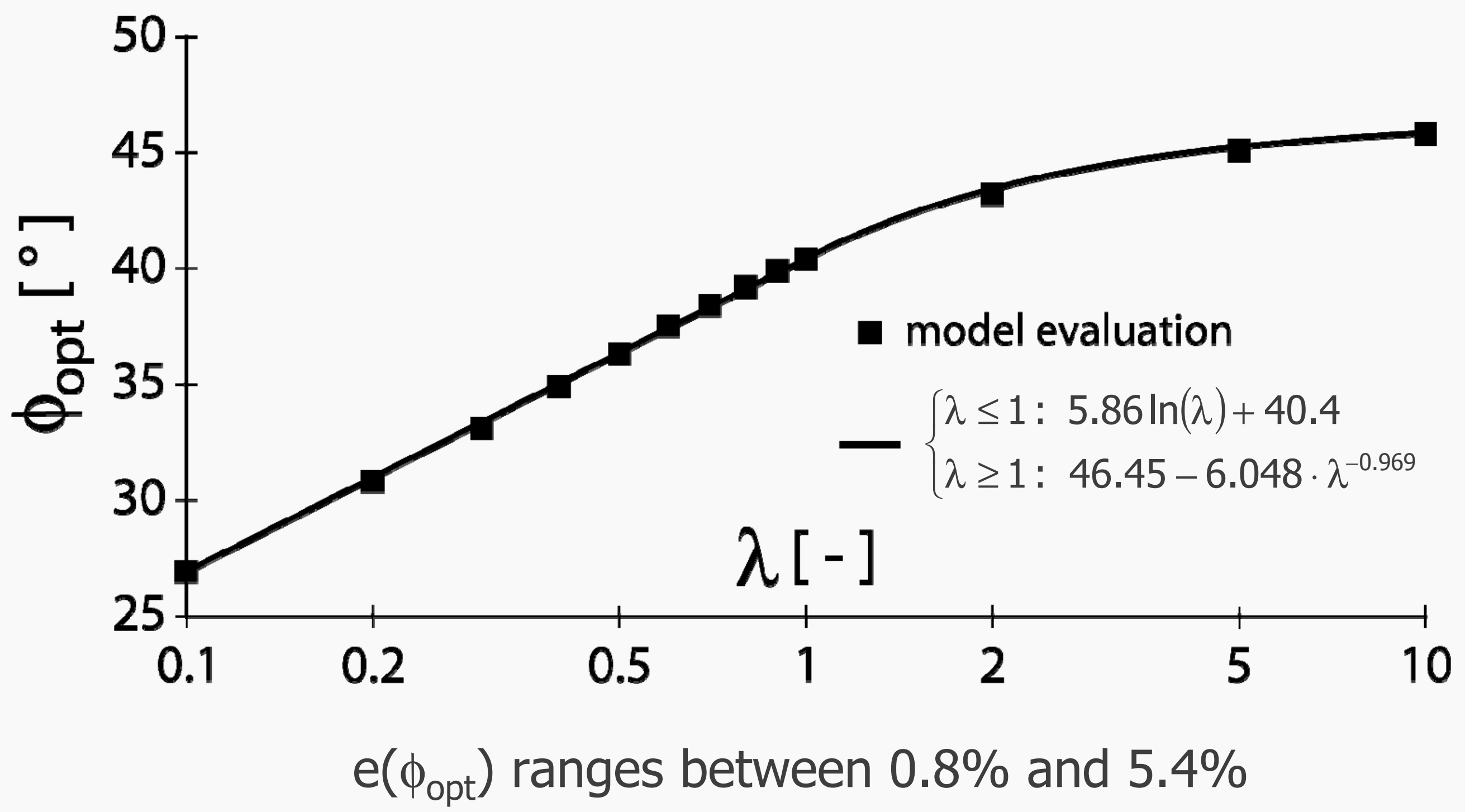




\section{Results}

Thermal impedance plots (1)

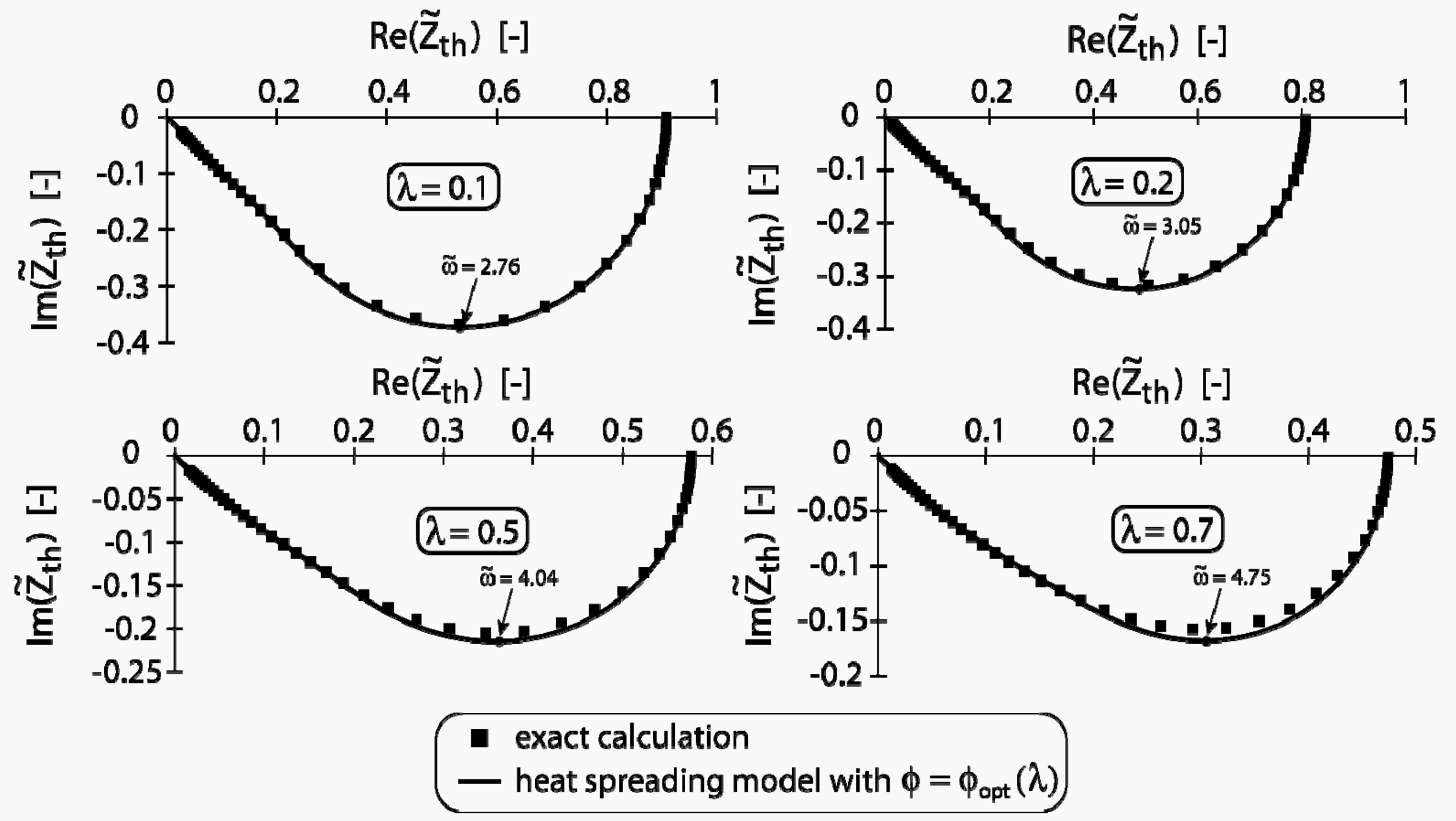




\section{Results}

\section{Thermal impedance plots (2)}

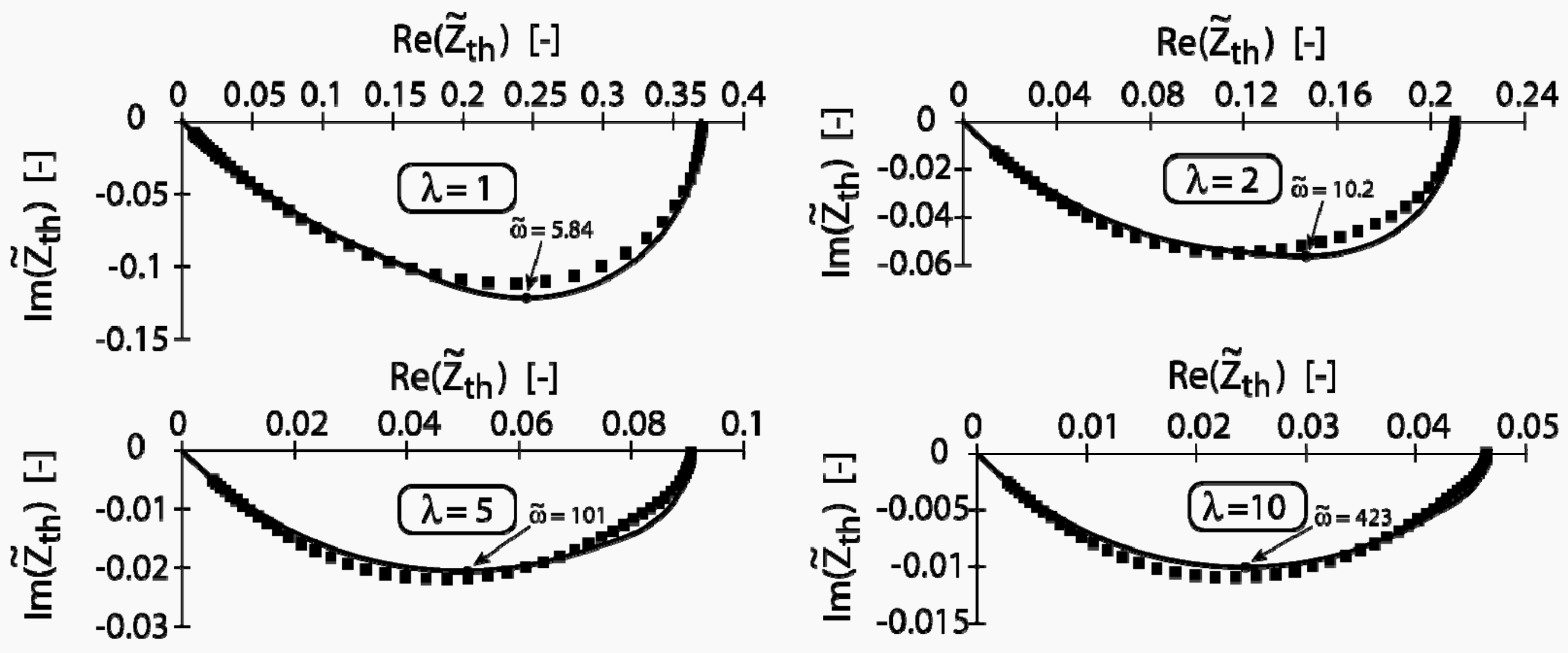

- exact calculation
- heat spreading model with $\phi=\phi_{\text {opt }}(\lambda)$ 


\section{Results}

\section{Step response}
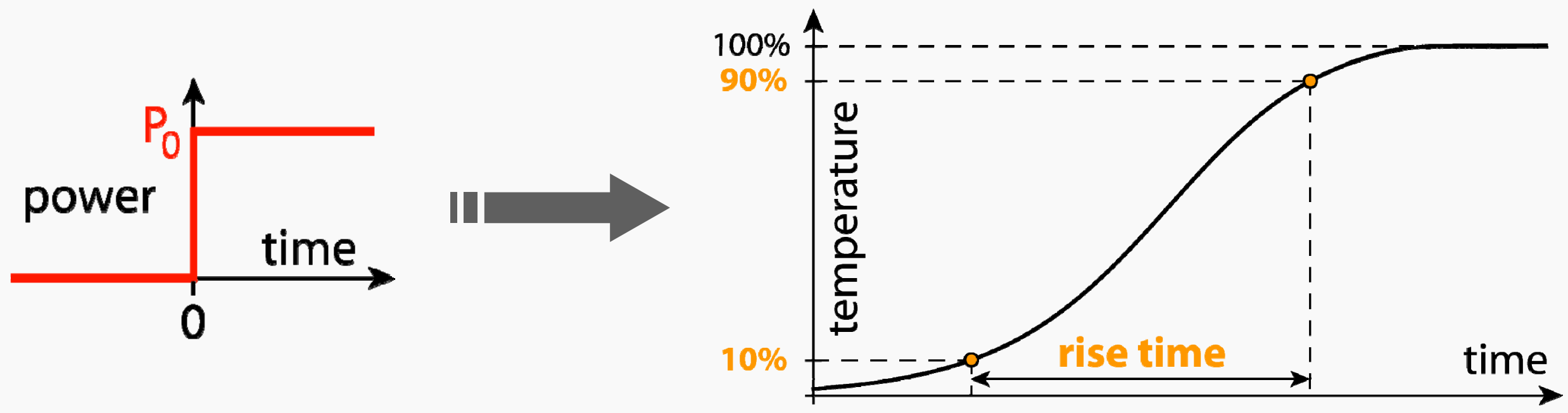

$P(f)=\frac{P_{0}}{2} \delta(f)+\frac{P_{0}}{j 2 \pi f}$

$$
T(t)=\int_{-\infty}^{\infty} Z_{t h}(f) P(f) \exp (j 2 \pi f t) d f
$$

$\tilde{T}(\tilde{\mathrm{t}})=\frac{1}{2}+\frac{1+2 \lambda \tan \phi}{\pi} \int_{0}^{\infty} \operatorname{Im}[\tilde{\mathrm{f}}[2 \lambda \tan \phi+j \tilde{\mathrm{f}} \operatorname{cotanh}(\tilde{\mathrm{j}} \tilde{\mathrm{j}} \tilde{\mathrm{f}})]] d \tilde{f}$

$$
\begin{gathered}
\mathrm{T}_{0}=\frac{\mathrm{P}_{0} \mathrm{Z}_{0}}{1+2 \lambda \tan \phi} \\
\mathrm{t}_{0}=\frac{2 \pi \mathrm{C}_{\mathrm{v}} \mathrm{t}_{\mathrm{s}}^{2}}{\mathrm{k}}
\end{gathered}
$$




\section{Results}

Transient heating curves

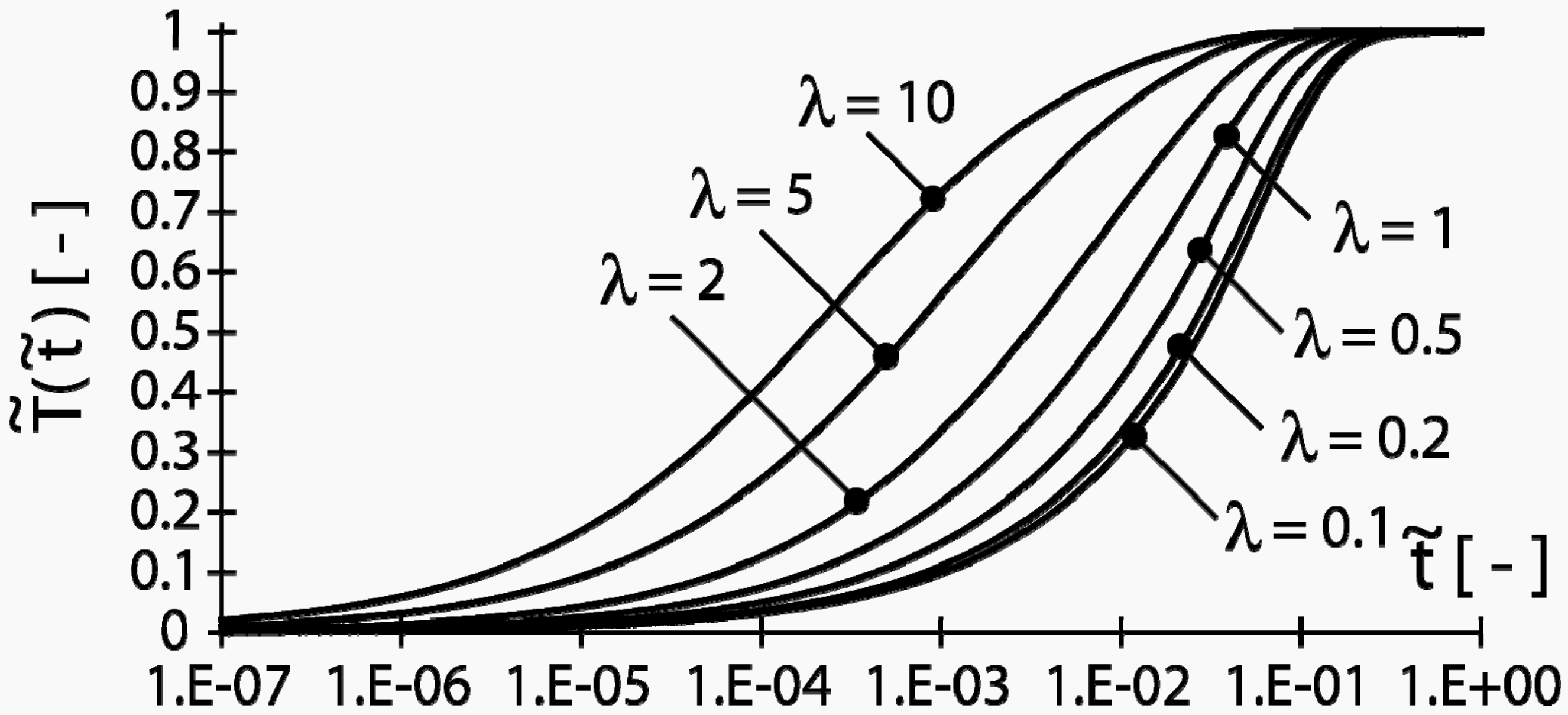




\section{Results}

Thermal rise time

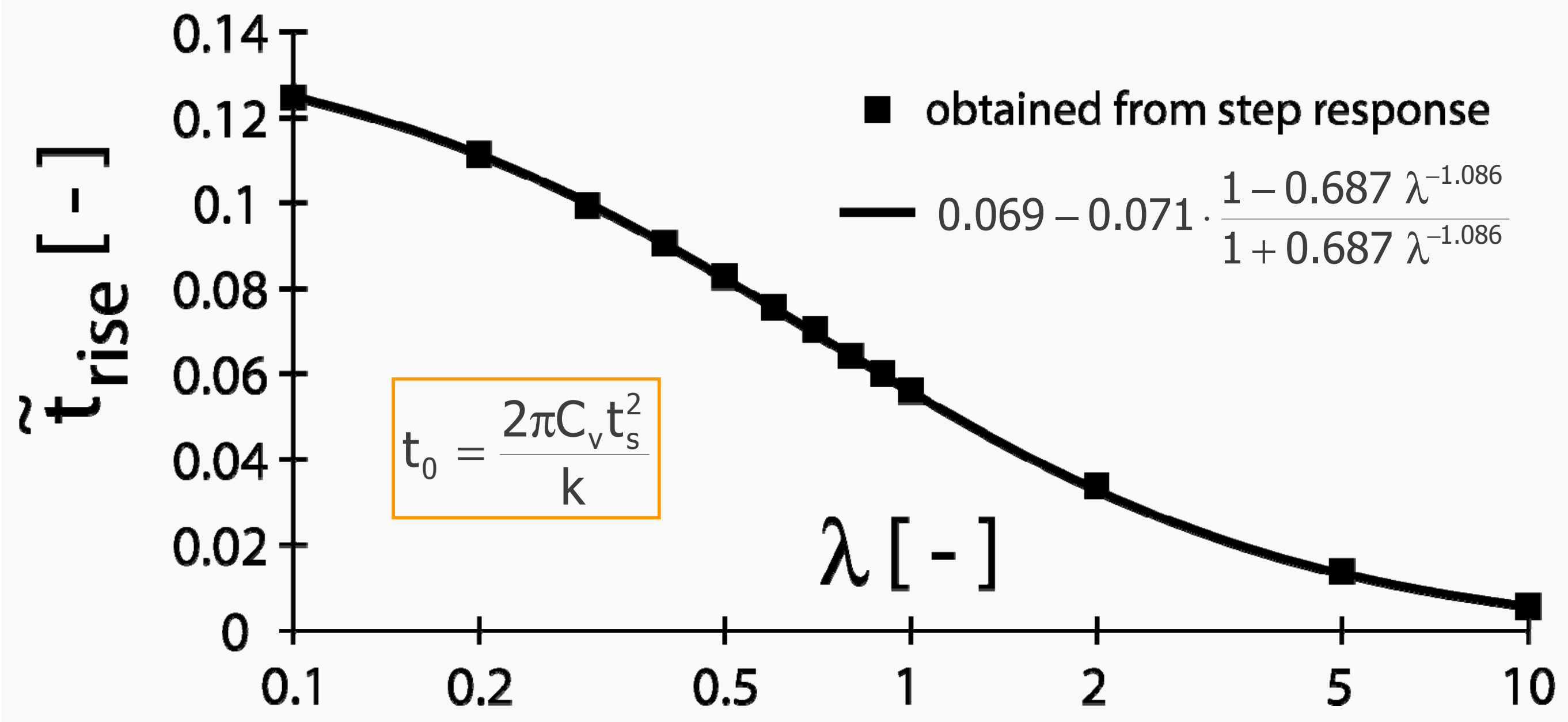




\section{Results}

Thermal rise time - denormalized

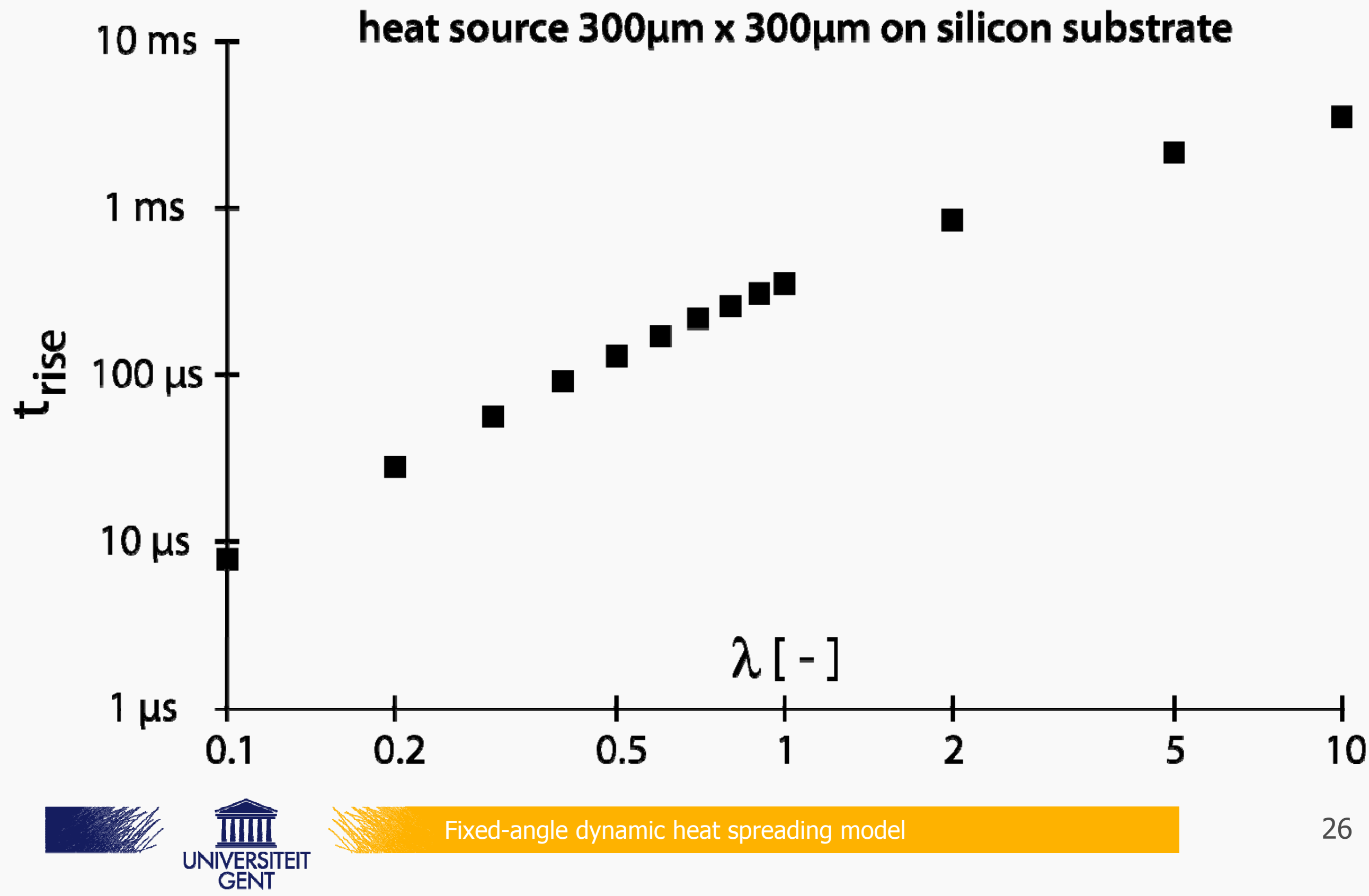




\section{Results}

\section{Global recipe \& case study}

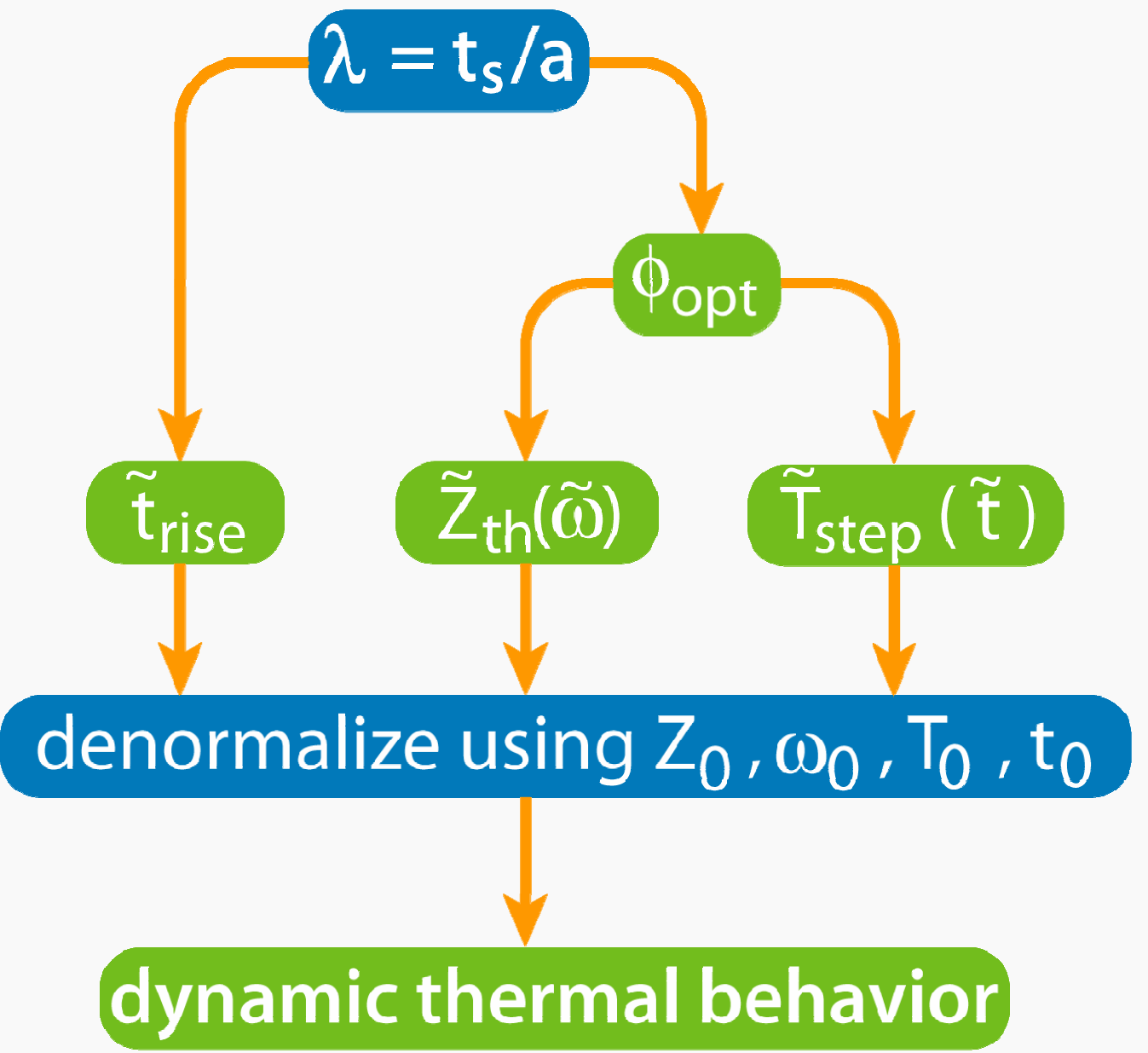

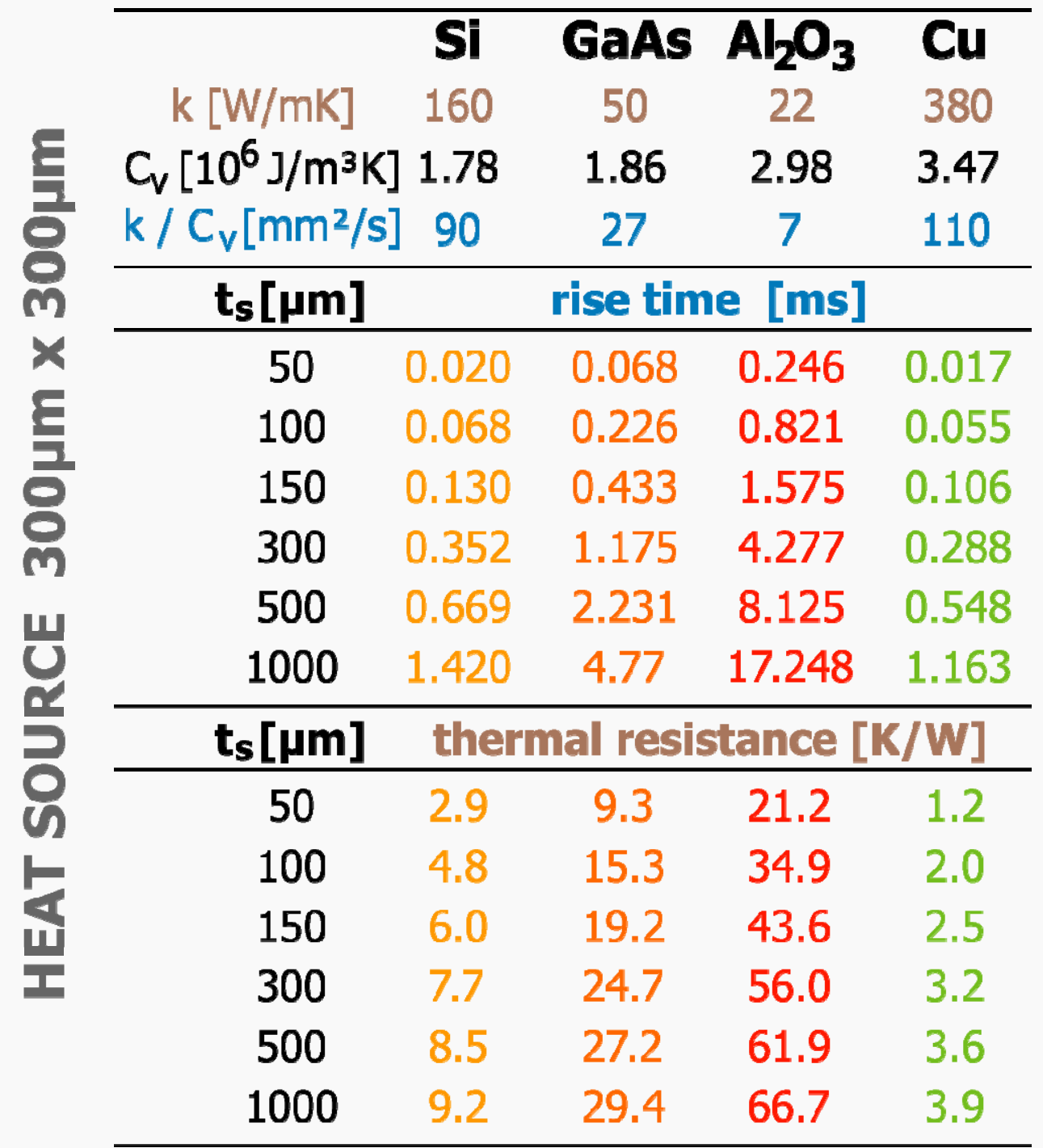




\section{Outline}

- Introduction

- Model definition

- Exact calculations

- Results

- Anisotropic substrates

- Conclusions 


\section{Anisotropic substrates}

Why?

- Inherent property of certain materials

- Thermal engineering: preferential heat flow
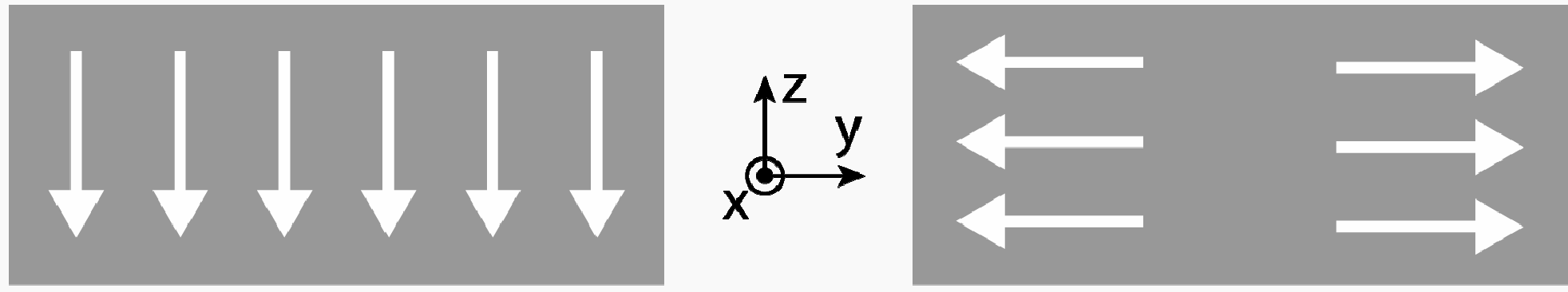

$$
\begin{aligned}
& k_{z}>k_{x y} \\
& \text { heat sink }
\end{aligned}
$$

chip

$$
\begin{gathered}
\mathbf{k}_{\mathbf{x y}}>\mathbf{k}_{\mathbf{z}} \\
\text { heat spreader }
\end{gathered}
$$

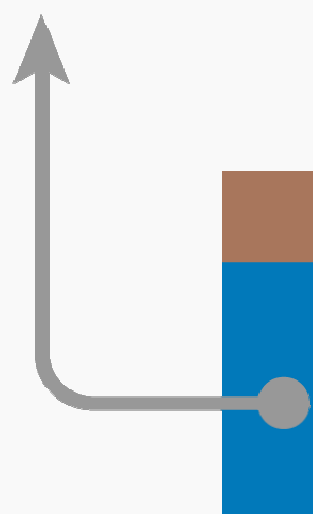

semiconductor die

solder (TIM)

thermal spreader

substrate 


\section{Anisotropic substrates \\ Practical example}

carbon fibres embedded in Al or Cu matrix

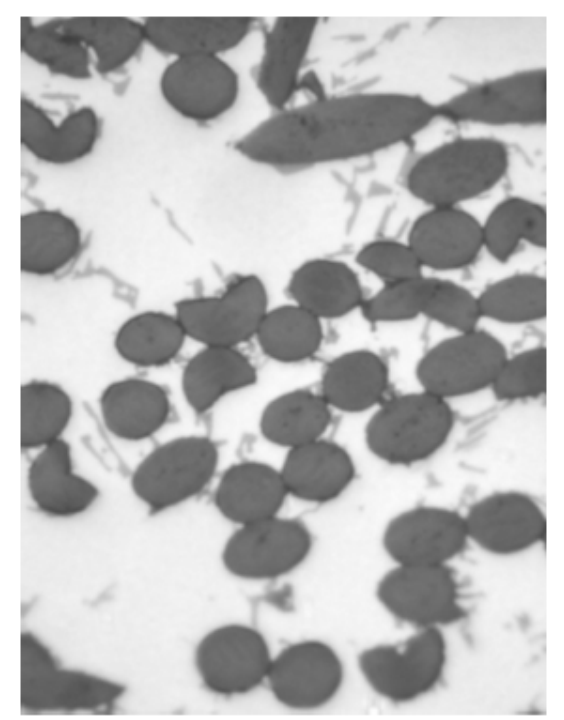

\section{In plane vs \\ $\mathbf{Z}$ direction}

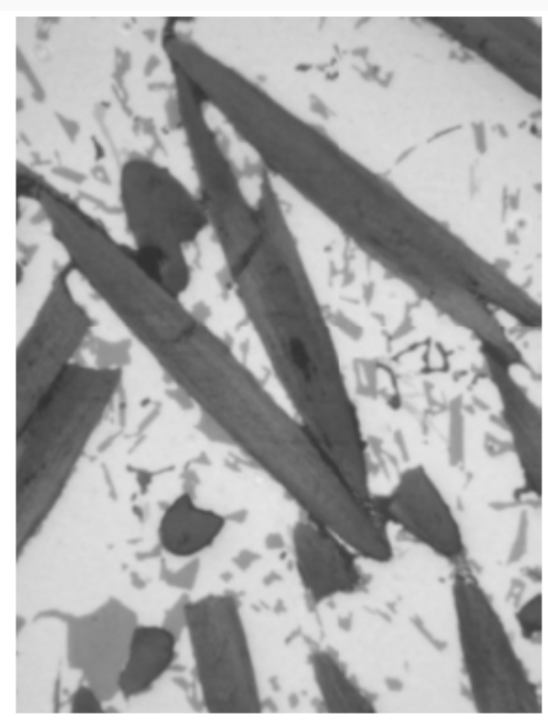

\begin{tabular}{|c|c|c|c|}
\hline $\begin{array}{c}\text { PHYSICAL \& MECHANICAL } \\
\text { PROPERTIES, (UNITS) }\end{array}$ & $\begin{array}{c}\text { Al filled } \\
\text { composite }\end{array}$ & $\begin{array}{r}\text { Cu filled } \\
\text { composite }\end{array}$ & NOTES \\
\hline Thermal Conductivity, (W/mK) & $260-300$ & $(300-340$ & In-plane values \\
\hline Thermal Conductivity, (W/mK) & $180-200$ & $220-250$ & Through-thickness values \\
\hline
\end{tabular}

(C) 2007 NovaPack Technologies - taken from Proc. IMAPS Workshop (31 Jan, La Rochelle)

$$
\widehat{\widehat{\text { UNIIIIII }}}
$$




\section{Anisotropic substrates Mathematical treatment (1)}

- 3D isotropic heat equation (phasor notation):

$$
k \nabla^{2} T(x, y, z)-j \omega C_{v} T(x, y, z)=0
$$

- if $\mathrm{k}_{\mathrm{xy}} \neq \mathrm{k}_{\mathrm{z}}$ :

$$
k_{x y} \frac{\partial^{2} T}{\partial x^{2}}+k_{x y} \frac{\partial^{2} T}{\partial y^{2}}+k_{z} \frac{\partial^{2} T}{\partial z^{2}}-j \omega C_{v} T=0
$$

- coordinate transformation: $z^{\prime}=\beta z$

$$
k_{x y} \frac{\partial^{2} T}{\partial x^{2}}+k_{x y} \frac{\partial^{2} T}{\partial y^{2}}+\beta^{2} k_{z} \frac{\partial^{2} T}{\partial z^{\prime 2}}-j \omega C_{v} T=0
$$

- Now choose $\beta$ such that $\beta^{2} k_{z}=k_{x y}$ 


\section{Anisotropic substrates Mathematical treatment (2)}

$-\beta=\sqrt{\frac{k_{x y}}{k_{z}}}$

"anisotropy factor"

$$
\mathrm{k}_{\mathrm{xy}} \nabla^{2} \mathrm{~T}\left(\mathrm{x}, \mathrm{y}, \mathrm{z}^{\prime}\right)-j \omega \mathrm{C}_{\mathrm{v}} \mathrm{T}\left(\mathrm{x}, \mathrm{y}, \mathrm{z}^{\prime}\right)=0
$$

- transformation of boundary conditions:

$-\left.\mathrm{k}_{\mathrm{z}} \frac{\partial \mathrm{T}}{\partial \mathrm{z}}\right|_{0}=\frac{\mathrm{P}}{\mathrm{a}^{2}} \Leftrightarrow-\mathrm{k}_{\mathrm{z}} \beta \frac{\partial \mathrm{T}}{\left.\partial \mathrm{z}^{\prime}\right|_{0}}=\frac{\mathrm{P}}{\mathrm{a}^{2}}$

$\Leftrightarrow \quad-\frac{k_{x y}}{\beta^{2}} \beta \frac{\partial T}{\left.\partial z^{\prime}\right|_{0}}=\frac{P}{a^{2}} \Leftrightarrow-k_{x y} \frac{\partial T}{\left.\partial z^{\prime}\right|_{0}}=\beta \frac{P}{a^{2}}$

Multiply dissipated power (and hence temperatures) with $\beta$

$$
\begin{gathered}
\mathrm{T}\left(\mathrm{z}=\mathrm{t}_{\mathrm{s}}\right)=0 \\
\Leftrightarrow \quad \mathrm{T}\left(\mathrm{z}^{\prime}=\beta \mathrm{t}_{\mathrm{s}}\right)=0
\end{gathered}
$$

Relate temperatures to appropriate location 


\section{Anisotropic substrates Model modification}

- temperature distribution in an anisotropic

$\left(k_{x y}, k_{z}\right)$ substrate with thickness $t_{s}$

$=$

$\beta$ times the temperature in an isotropic

$\left(k_{x y}\right)$ substrate with thickness $\beta t_{s}$.

- (!!!) keep factor $\beta$ in $Z_{\text {th }}$ : related to original source

- Hence:

$$
\tilde{Z}_{\text {th }}^{\prime}(\lambda, \phi)=\beta \cdot \tilde{Z}_{\text {th }}\left(\beta \lambda, \phi^{\prime}\right) \quad \text { with } \quad \phi^{\prime}=\phi_{\text {opt }}(\beta \lambda)
$$

$$
\tilde{\mathrm{t}}_{\text {rise }}^{\prime}(\lambda)=\tilde{\mathrm{t}}_{\text {ise }}(\beta \lambda)
$$

- (!!!) Don’t forget to reinterpret normalization values 


\section{Anisotropic substrates}

\section{Impact on steady state}

- $\mathrm{R}_{\mathrm{th}}=\frac{\mathrm{r}_{0} \mathrm{t}_{\mathrm{s}}}{1+\alpha \mathrm{t}_{\mathrm{s}}}=\frac{\mathrm{t}_{\mathrm{s}}}{\mathrm{ka}^{2}} \cdot \frac{1}{1+2 \lambda \tan \phi} \quad \mathbf{k}=\mathbf{k}_{\mathbf{x y}}=$ constant

$$
\longrightarrow \frac{\mathrm{R}^{\prime}{ }_{\text {th }}}{\mathrm{R}_{\text {th }}}=\frac{\mathrm{R}}{\mathrm{R}_{\text {iso }}}=\beta^{2} \cdot \frac{1+2 \lambda \tan \left(\phi_{\text {opt }}(\lambda)\right)}{1+2 \beta \lambda \tan \left(\phi_{\text {opt }}(\beta \lambda)\right)}
$$

- intuitive expectations (based on 1-D viewpoint)

" $\mathrm{k}_{\mathrm{z}}$ is dominant, pretend isotropic"

$$
\mathrm{R}_{\mathrm{th}} \div \frac{1}{\mathrm{k}} \rightarrow \frac{\mathrm{R}}{\mathrm{R}_{\text {iso }}}=\frac{1 / \mathrm{k}_{\mathrm{z}}}{1 / \mathrm{k}_{\mathrm{xy}}}=\beta^{2}
$$

"anistropic: $\beta$ times temp. in isotropic substrate $\mathrm{k}_{\mathrm{xy}}$ but $\beta$ times thicker"

$$
\mathrm{R}_{\text {th }} \div \mathrm{t}_{\mathrm{s}} \longrightarrow \frac{\mathrm{R}}{\mathrm{R}_{\text {iso }}}=\beta^{2}
$$




\section{Anisotropic substrates \\ $\mathbf{R}_{\mathrm{th}} @$ constant $\mathbf{k}_{\mathrm{xy}}$ : results}

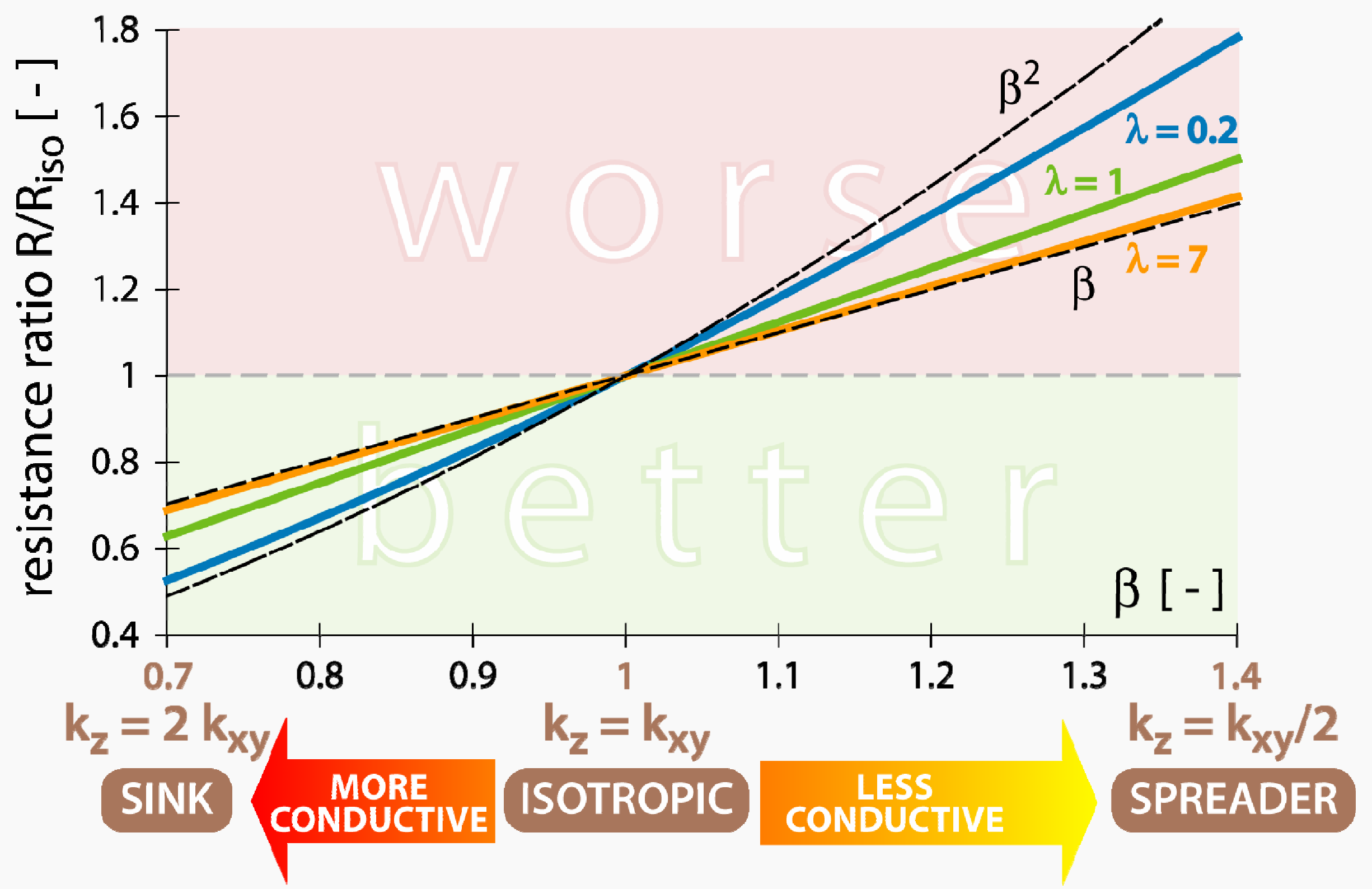




\section{Anisotropic substrates \\ $\mathbf{R}_{\mathrm{th}} @$ constant $\mathbf{k}_{\mathrm{xy}}$ : analysis}

\section{THIN SUBSTRATES}

$\beta^{2}$ reasonable for $R / R_{\text {iso }}$

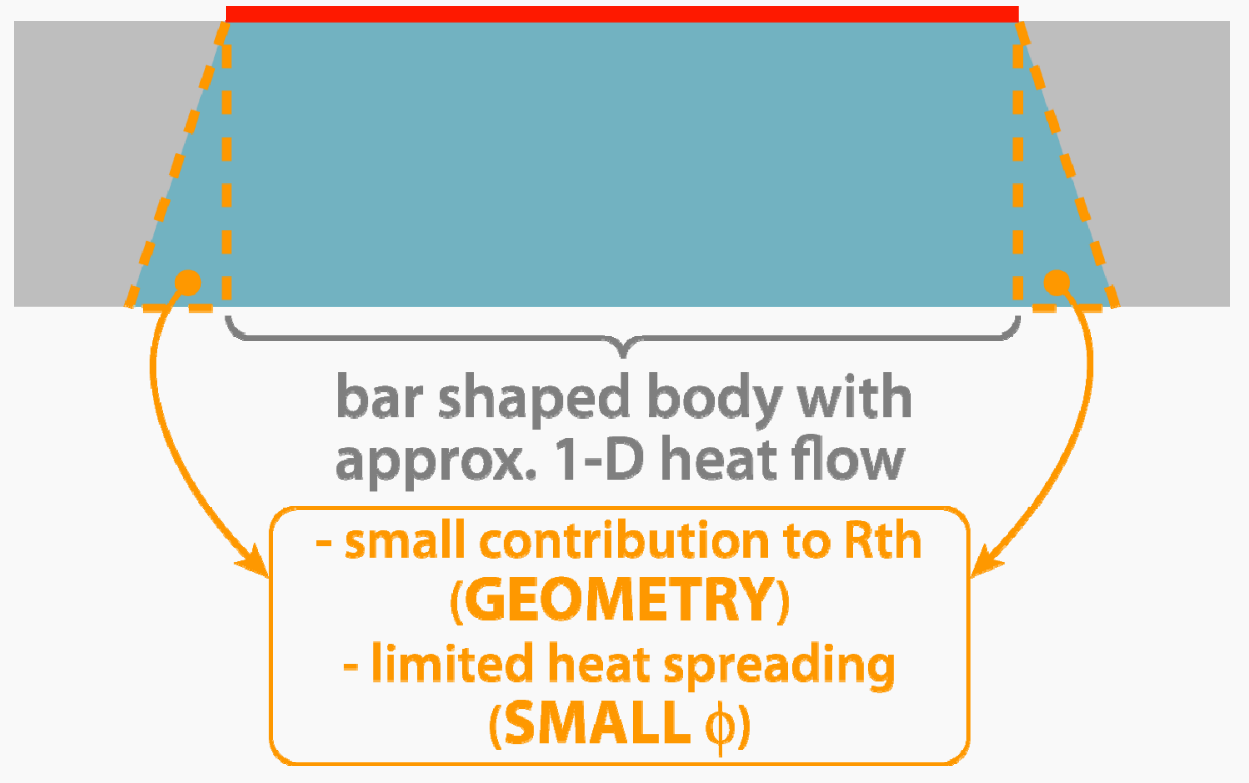

$$
\frac{\mathrm{R}}{\mathrm{R}_{\text {iso }}} \approx \frac{\frac{\mathrm{t}_{\mathrm{s}}}{\mathrm{k}_{\mathrm{z}} \mathrm{a}^{2}}}{\frac{\mathrm{t}_{\mathrm{s}}}{\mathrm{k}_{\mathrm{xy}} \mathrm{a}^{2}}}=\frac{\mathrm{k}_{\mathrm{xy}}}{\mathrm{k}_{\mathrm{z}}}=\beta^{2}
$$

THICK SUBSTRATES

$$
\mathrm{R} / \mathrm{R}_{\text {iso }} \approx \beta
$$

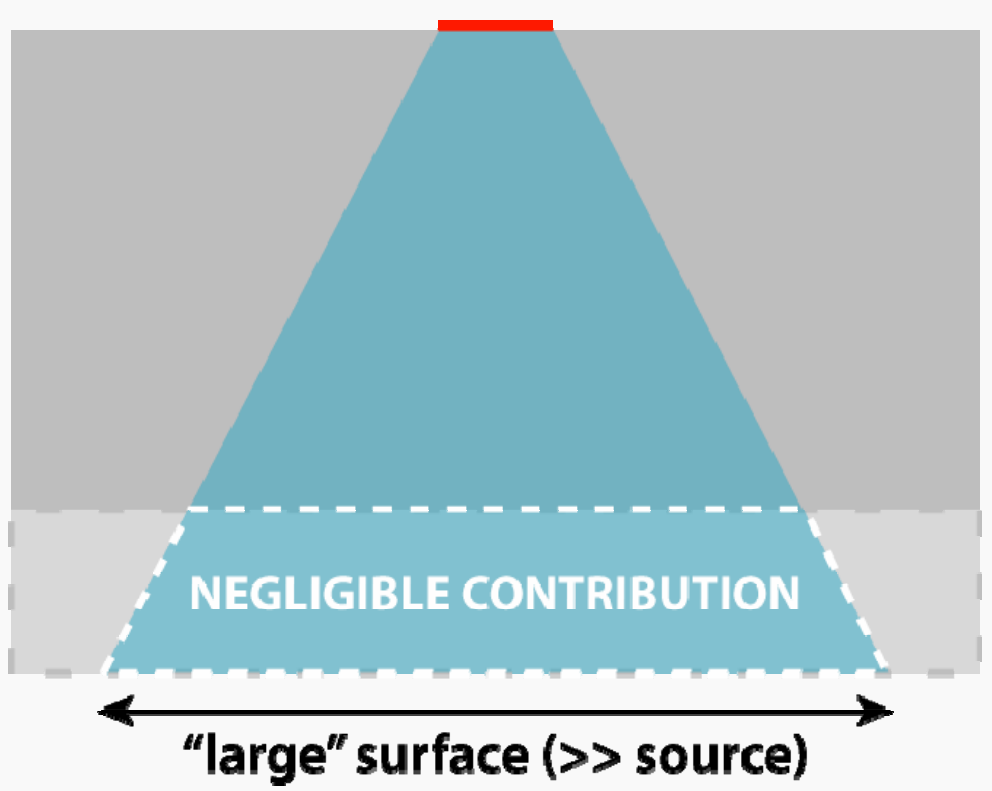

$\mathrm{R}=\beta \cdot \mathrm{R}_{\text {scaled pyr }} \approx \beta \cdot \mathrm{R}_{\text {iso }}$ 


\section{Anisotropic substrates Impact on transient behaviour}

- temperature distribution in an anisotropic

$\left(\mathrm{k}_{\mathrm{xy}}, \mathrm{k}_{\mathrm{z}}\right)$ substrate with thickness $\mathrm{t}_{\mathrm{s}}$ $=$

$\beta$ times the temperature in an isotropic $\left(\mathrm{K}_{\mathrm{xy}}\right)$ substrate with thickness $\beta \mathrm{t}_{\mathrm{s}}$.

no influence on trise: time scale unaltered

$$
\longrightarrow \tilde{\mathrm{t}}_{\text {rise }}^{\prime}(\lambda)=\tilde{\mathrm{t}}_{\text {ise }}(\beta \lambda) \quad \mathrm{t}_{0}=\frac{2 \pi \mathrm{C}_{\mathrm{v}} \mathrm{t}_{\mathrm{s}}^{2}}{\mathrm{k}}
$$

$$
\frac{\mathrm{t}_{\text {rise }}^{\prime}}{\mathrm{t}_{\text {rise }}}=\frac{\Delta}{\Delta_{\text {iso }}}=\beta^{2} \cdot \frac{0.069-0.071 \cdot \frac{1-0.687(\beta \lambda)^{-1.086}}{1+0.687(\beta \lambda)^{-1.086}}}{0.069-0.071 \cdot \frac{1-0.687 \cdot \lambda^{-1.086}}{1+0.687 \cdot \lambda^{-1.086}}}
$$

$k=k_{x y}=$ constant 


\section{Anisotropic substrates \\ $\mathbf{t}_{\text {rise }} @$ constant $\mathbf{k}_{\mathrm{xy}}:$ results}

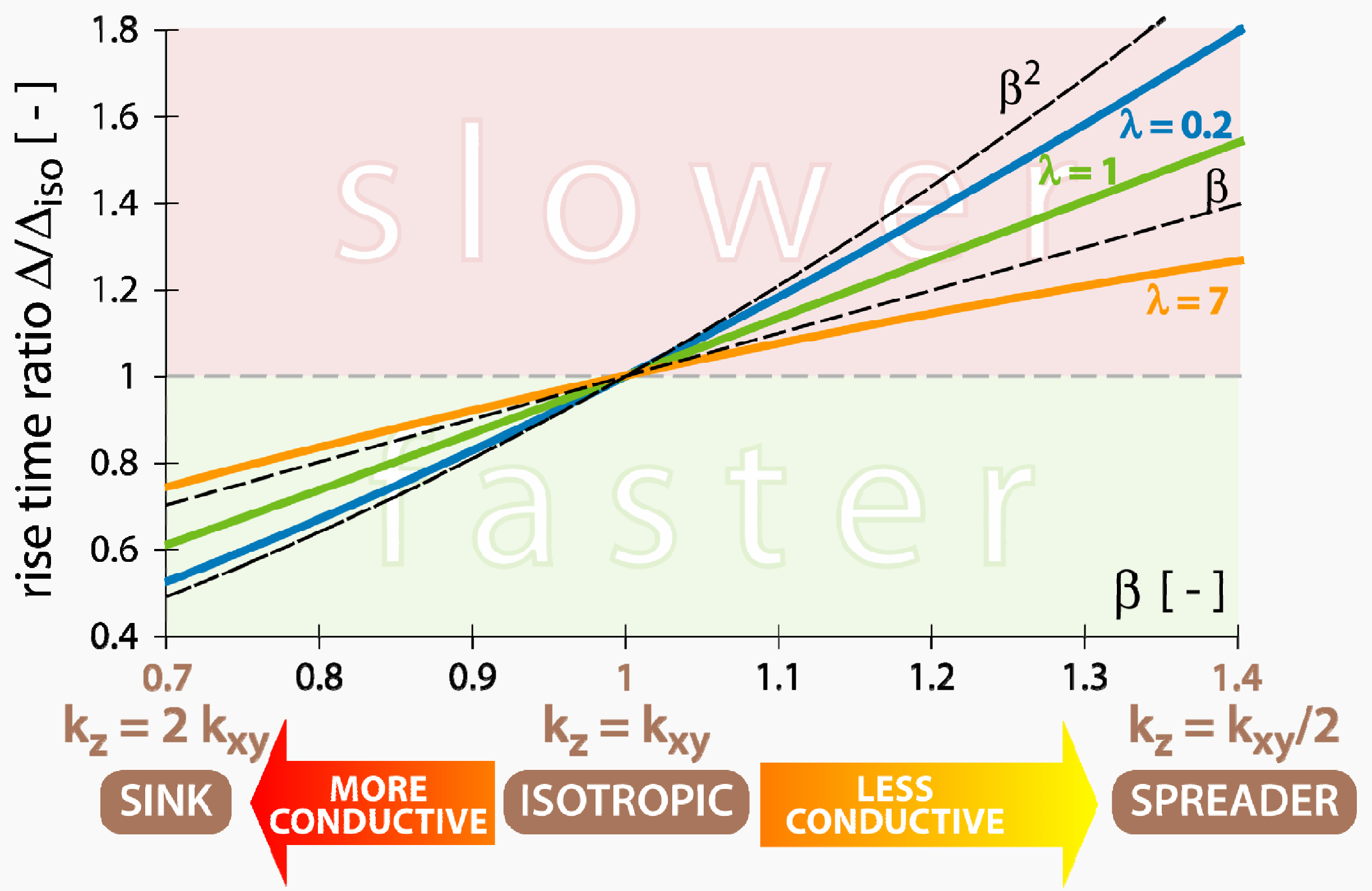




\section{Anisotropic substrates $t_{\text {rise }} @$ constant $k_{x y}$ : analysis (1)}

THIN SUBSTRATES: $\beta^{2}$ reasonable for $\Delta / \Delta_{\text {iso }}$

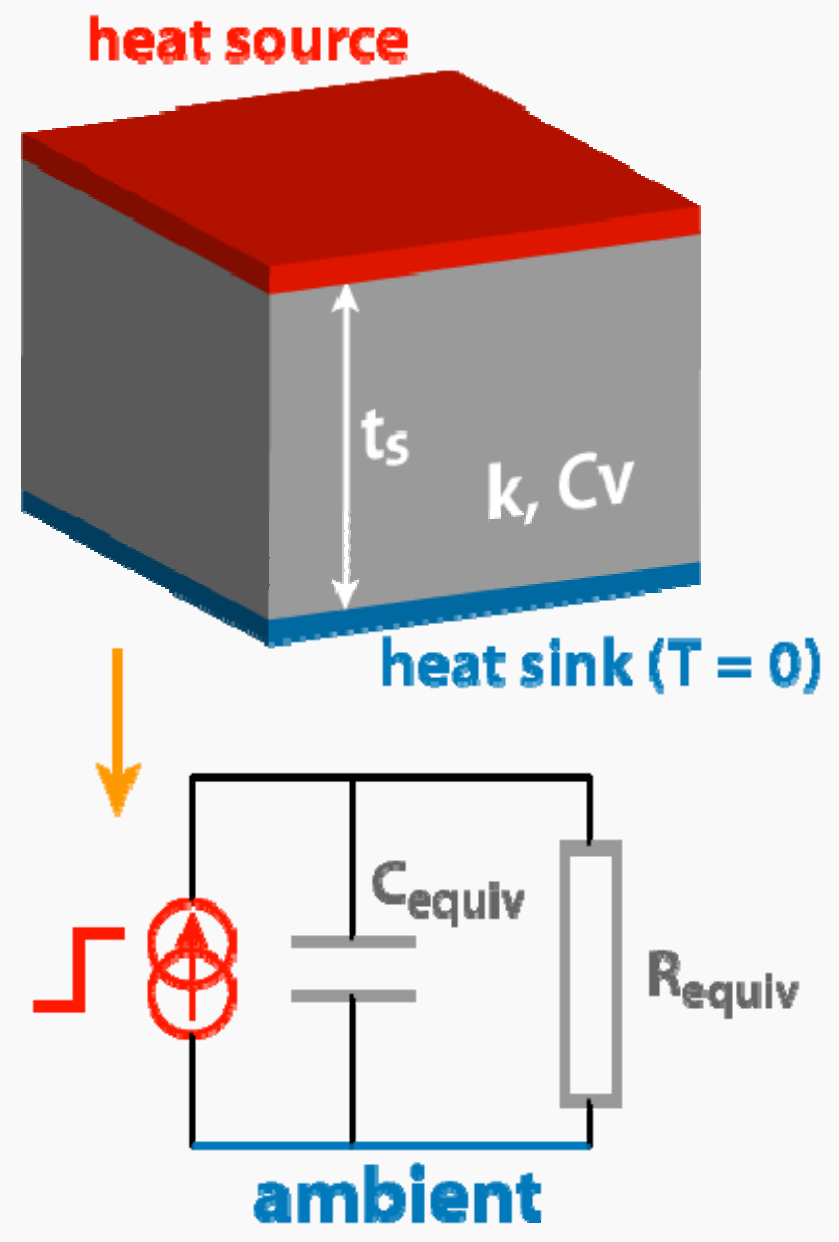
step response for 1-D heat flow

$$
\begin{aligned}
\tilde{T} & =1-\exp \left(-t / \tau_{\text {equiv }}\right) \\
\tau_{\text {equiv }} & =R_{\text {equiv }} C_{\text {equiv }} \approx 0.369 \frac{\mathrm{C}_{\mathrm{v}} \mathrm{t}_{\mathrm{s}}^{2}}{\mathrm{k}}
\end{aligned}
$$

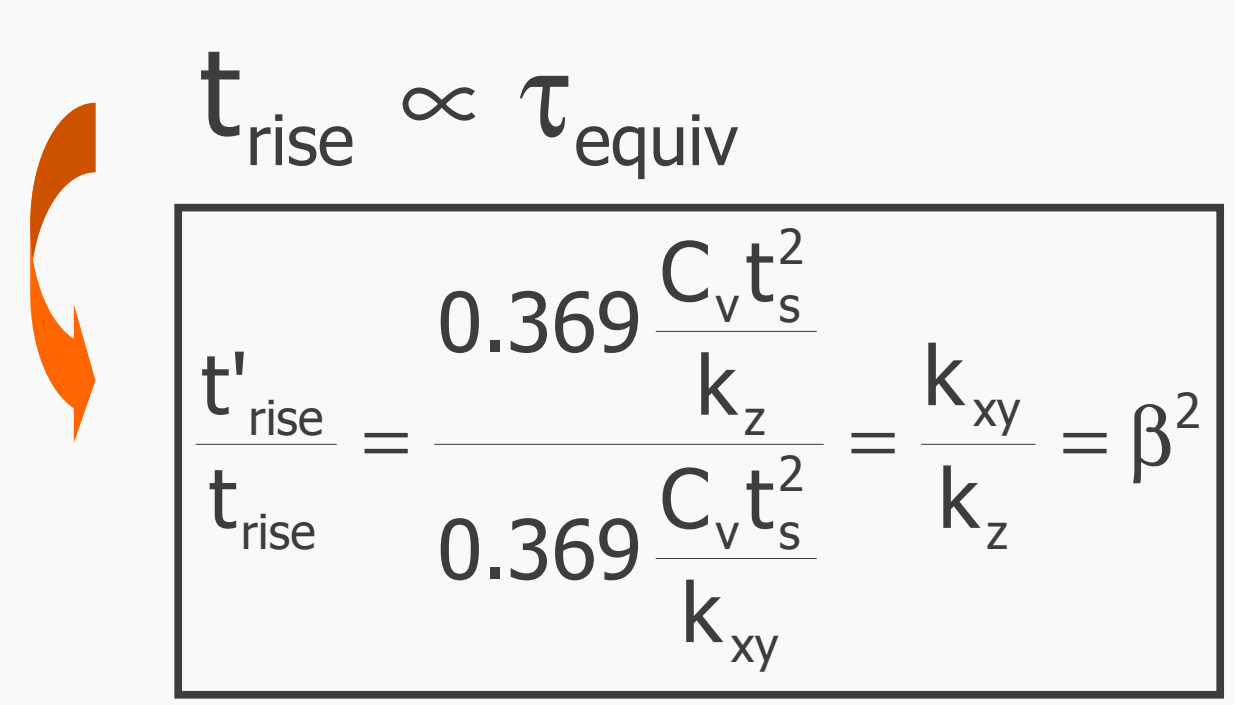

- 


\section{Anisotropic substrates $\mathbf{t}_{\text {rise }} @$ constant $\mathbf{k}_{\mathrm{xy}}:$ analysis (2)}

- OTHER CASES: physical explanation not straightforward at all

- thermal diffusion characterized by whole spectrum of time constants (even for 1-D heat flow)

- trouble:

- fitting with single exponential impossible if no dominant time constant

- $\mathrm{C}_{\text {equiv }}$ not directly proportional to material volume $-\ldots$ 


\section{Outline}

- Introduction

- Model definition

- Exact calculations

- Results

- Anisotropic substrates

- Conclusions 


\section{Conclusions}

- dynamic fixed-angle heat spreading model

- extends numerous works for steady state

- frequency domain representation $\left(Z_{\mathrm{th}}\right)$ \& step response

- error $<5 \%$ if appropriate spreading angle chosen

- simple expressions for $\phi_{\text {opt }} \& t_{\text {rise }}$

- valid for wide range of thicknesses $(\lambda=0.1 \ldots 10)$

- can be applied for anisotropic materials

- effect of anisotropy quantified by $\beta+$ physically explained

- allows quick yet accurate estimation of time-dependent thermal behaviour 


\section{Acknowledgements}

I wish to thank

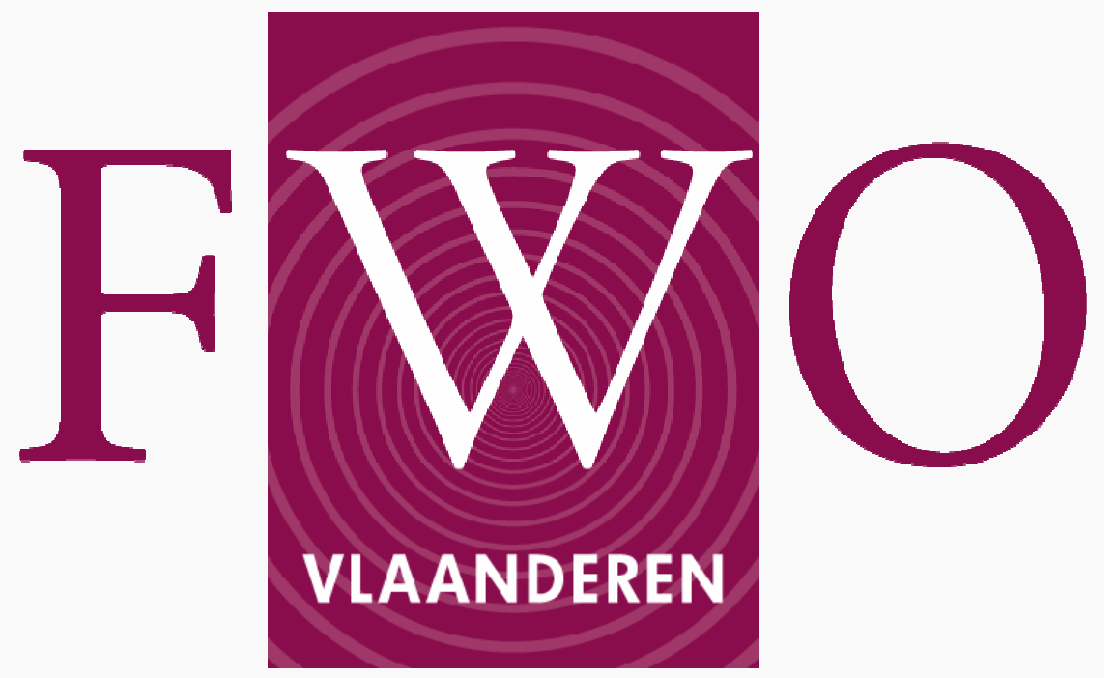

Research Foundation - Flanders

for supporting the presented work. 